\title{
Unique properties of thymic antigen-presenting cells promote epigenetic imprinting of alloantigen-specific regulatory $\mathbf{T}$ cells
}

\author{
Garima Garg ${ }^{1}$, Eirini Nikolouli ${ }^{1}$, Matthias Hardtke-Wolenski ${ }^{2}$, Aras Toker ${ }^{1}$, Naganari \\ Ohkura $^{3,4}$, Michael Beckstette ${ }^{1}$, Takahisa Miyao ${ }^{5}$, Robert Geffers ${ }^{6}$, Stefan Floess ${ }^{1}$, \\ Norbert Gerdes 7,8 , Esther Lutgens ${ }^{7,9}$, Anke Osterloh ${ }^{10}$, Shohei Hori ${ }^{5}$, Shimon \\ Sakaguchi ${ }^{3,4}$, Elmar Jaeckel ${ }^{2}$ and Jochen Huehn ${ }^{1}$ \\ ${ }^{1}$ Department of Experimental Immunology, Helmholtz Centre for Infection Research, Braunschweig, Germany \\ 2 Department of Gastroenterology, Hepatology, Endocrinology, Hannover Medical School, Hannover, Germany \\ 3 Department of Experimental Immunology, World Premier International Immunology Frontier Research Center, Osaka \\ University, Suita, Japan \\ ${ }^{4}$ Department of Experimental Pathology, Institute for Frontier Medical Sciences, Kyoto University, Kyoto, Japan \\ ${ }^{5}$ Laboratory for Immune Homeostasis, RIKEN Center for Integrative Medical Sciences, Yokohama City, Kanagawa, Japan \\ ${ }^{6}$ Genome Analytics, Helmholtz Centre for Infection Research, Braunschweig, Germany \\ ${ }^{7}$ Institute for Cardiovascular Prevention, Ludwig-Maximilians-University, Munich, Germany \\ ${ }^{8}$ Division of Cardiology, Pulmonology, and Vascular Medicine Medical Faculty, University Hospital Düsseldorf, Düsseldorf, \\ Germany \\ ${ }^{9}$ Department of Medical Biochemistry, Academic Medical Center, University of Amsterdam, AZ, Amsterdam, The Netherlands \\ ${ }^{10}$ Department of Immunology, Bernhard Nocht Institute for Tropical Medicine, Hamburg, Germany \\ Correspondence to: Jochen Huehn, email: jochen.huehn@helmholtz-hzi.de \\ Keywords: regulatory T cells, thymic APCs, epigenetic modification, alloantigen-specificity, Immunology and Microbiology Section, \\ Immune response, Immunity \\ Received: October 15, $2016 \quad$ Accepted: March 03, $2017 \quad$ Published: March 15, 2017
}

Copyright: Garg et al. This is an open-access article distributed under the terms of the Creative Commons Attribution License (CC-BY), which permits unrestricted use, distribution, and reproduction in any medium, provided the original author and source are credited.

\section{ABSTRACT}

Regulatory T cells (Tregs) are potential immunotherapeutic candidates to induce transplantation tolerance. However, stability of Tregs still remains contentious and may potentially restrict their clinical use. Recent work suggested that epigenetic imprinting of Foxp3 and other Treg-specific signature genes is crucial for stabilization of immunosuppressive properties of Foxp $3^{+}$Tregs, and that these events are initiated already during early stages of thymic Treg development. However, the mechanisms governing this process remain largely unknown. Here we demonstrate that thymic antigen-presenting cells (APCs), including thymic dendritic cells (t-DCs) and medullary thymic epithelial cells ( $\mathrm{MTECs}$ ), can induce a more pronounced demethylation of Foxp3 and other Treg-specific epigenetic signature genes in developing Tregs when compared to splenic DCs (sp-DCs). Transcriptomic profiling of APCs revealed differential expression of secreted factors and costimulatory molecules, however neither addition of conditioned media nor interference with costimulatory signals affected Foxp3 induction by thymic APCs in vitro. Importantly, when tested in vivo both $\mathbf{m T E C}$ - and t-DC-generated alloantigen-specific Tregs displayed significantly higher efficacy in prolonging skin allograft acceptance when compared to Tregs generated by sp-DCs. Our results draw attention to unique properties of thymic APCs in initiating commitment towards stable and functional Tregs, a finding that could be highly beneficial in clinical immunotherapy.

\section{INTRODUCTION}

$\mathrm{CD}^{+}$regulatory T cells (Tregs) - characterized by the expression of the lineage specification factor Foxp3
- play an indispensable role for the maintenance of immune homeostasis and self-tolerance [1]. In addition, Foxp $3^{+}$Tregs also represent a cell type that is highly relevant for clinical considerations, e.g. for the treatment 
of autoimmune conditions, graft-versus-host disease (GvHD) and allograft rejection [2-7], and the efficiency of Tregs in decreasing the incidence of acute GvHD in first clinical trials have encouraged further use of these cells in cellular therapies [8]. Foxp $3^{+}$Tregs can be generated in large numbers in vitro by stimulating conventional $\mathrm{CD}^{+} \mathrm{T}$ cells in presence of TGF- $\beta$ (in vitro induced Tregs, iTregs) [9], but stability of Foxp3 expression and suppressive potential of these cells after adoptive transfer is discussed controversially [10-13]. Thus, generation of Tregs with a stable immunosuppressive phenotype is crucial to render their use in therapeutic approaches feasible [14]. This is especially true in case of adoptive Treg therapy for autoimmune diseases and after transplantation which will likely require the use of antigen-specific Tregs that could turn into detrimental effector cells if the regulatory phenotype is lost.

Stability of Foxp3 expression correlates with DNA demethylation at a conserved intronic CpG-rich region within the Foxp3 locus, designated Treg-specific demethylated region (TSDR) [15]. Demethylation at the TSDR (also known as CNS2) is not required for initiation of Foxp3 expression, but for its long-term maintenance $[10,16,17]$. However, stable Foxp3 expression is not sufficient for fully functional Tregs. Instead, selective demethylation of a number of Treg-specific signature genes including Ctla4, Gitr, Eos and Helios in addition to the TSDR is crucially required for Foxp $3^{+} \mathrm{T}$ cells to acquire Treg-specific gene expression, lineage stability and full suppressive activity [13, 18, 19]. Accordingly, iTregs with fully methylated Treg-specific epigenetic signature genes display only instable Foxp3 expression and lack suppressive capacity upon adoptive transfer in vivo [10, 11, 13]. Hence, understanding those mechanisms that cause selective demethylation of Treg-specific epigenetic signature genes in developing Tregs could open up ways to manipulate the DNA methylation status of Tregs and allow safe application of in vitro generated Tregs for therapeutic approaches [20].

Although it is known that selective demethylation of the TSDR and other Treg-specific epigenetic signature genes is initiated already during early stages of thymic Treg development $[13,21,22]$, cellular players and molecular mechanisms governing epigenetic imprinting of the Treg fate within the thymus remain largely enigmatic. It is tempting to speculate that thymic antigen-presenting cells (APCs) are involved in this process since thymic Treg development requires CD4SP (CD4 single positive) thymocytes encountering their cognate antigen presented by thymic APCs together with proper costimulation and cytokine signaling [23-36].

To investigate the role of thymic APCs, we focused on medullary thymic epithelial cells (mTECs) and thymic dendritic cells (t-DCs), those APCs that are predominantly found in the thymic medulla, the main hub of Foxp $3^{+}$Treg development [37]. We demonstrate that both mTECs and t-DCs not only have a preferential ability to induce alloantigen-specific Foxp $3^{+}$Tregs in vitro (allo-iTregs) when compared to splenic DCs (sp-DCs), but also to initiate a more pronounced demethylation of the TSDR and other Treg-specific epigenetic signature genes. Transcriptomic profiling of APCs uncovered differential expression of numerous immunologically relevant molecules, however neither secreted factors nor major costimulatory signals seemed to be functionally relevant for induction of Foxp3 ${ }^{+}$Tregs in in vitro allogeneic cultures. Importantly, thymic APC-induced allo-iTregs showed a superior suppressive capacity when tested in a highly immunogenic, allogeneic skin transplantation model. Viewed as a whole, our results demonstrate that thymic APCs harbor unique properties, which are instrumental for the epigenetic imprinting and stabilization of Foxp $3^{+}$Tregs.

\section{RESULTS}

\section{Thymic APC-induced Foxp3 $^{+}$Tregs show highest demethylation at Treg-specific epigenetic signature genes}

Previous studies have demonstrated that selective demethylation of the TSDR and other Treg-specific epigenetic signature genes is initiated already during early stages of thymic Treg development [13, 21, 22]. To investigate the specific contribution of thymic APCs to this process, an alloantigen-specific in vitro stimulation system was established. As stimulators, we chose APCs from the medulla since this region is the major site of thymic Foxp $3^{+}$ Treg development [37]. CD45-EpCAM ${ }^{+}$Ly51' ${ }^{-}$TECs and CD $45^{+} \mathrm{CD} 11 \mathrm{c}^{\text {hi }}$ Lin $^{-} \mathrm{DCs}$ (t-DCs) were isolated ex vivo from thymi of $\mathrm{BALB} / \mathrm{c}$ mice, and $\mathrm{CD} 45^{+} \mathrm{CD} 11 \mathrm{c}^{\mathrm{hi}} \mathrm{Lin}^{-}$ DCs from spleens (sp-DCs) of BALB/c mice were taken as controls (sp-DCs) (Supplementary Figure S1A). As responder cells, CD4SP Foxp3- thymocytes were purified from Foxp3 reporter mice (C57BL/6 background) and cultured with mTECs, t-DCs or sp-DCs for six days in presence of exogenous IL-2, resulting in a substantial and comparable proliferation of responder cells in all cultures (data not shown). First, we studied the capacity of the different APCs to induce Foxp3 expression in the responder cells. Flow cytometric analysis revealed that both mTECs and t-DCs had induced significantly higher frequencies of Foxp3 $3^{+}$allo-iTregs as compared to sp-DCs (Figure 1A, 1B). Importantly, the same optimal ratio of APCs to thymocytes was used for t-DCs and sp-DCs (1:10), while mTECs induced maximal percentages of allo-iTregs at a ratio of 1:50 (Supplementary Figure S1B). Hence, for all further allo-iTreg induction experiments these optimal ratios were used. As expected from a previous report [38], peripheral $\mathrm{CD}^{+}{ }^{+}$Foxp $^{-} \mathrm{T}$ cells 
isolated from spleen and lymph nodes failed to efficiently differentiate into allo-iTregs upon coculture with thymic APCs (Supplementary Figure S1C). However, when CD4SP Foxp3- thymocytes, containing CD25+Foxp3- Treg precursors [23-36], were cultured with syngeneic APCs for six days in presence of exogenous IL-2 as described previously [28], we also observed that both mTECs and t-DCs had induced higher frequencies of Foxp $3^{+}$Tregs as compared to sp-DCs (Supplementary Figure S1D). Thus, CD4SP Foxp3- thymocytes displaying an intrinsically high ability to develop into Foxp $3^{+}$Tregs were chosen for all future experiments.

Having shown that thymic APCs have a higher capacity to induce Foxp $3^{+}$allo-iTregs as compared to sp-DCs, we next asked for the methylation status of the Treg-specific epigenetic signature genes in these Foxp $3^{+}$ allo-iTregs. Genomic DNA was isolated from sort-purified Foxp $^{+}$allo-iTregs, bisulfite treated and analyzed by pyrosequencing. Notably t-DC-induced, but also mTECinduced allo-iTregs showed a pronounced demethylation of the TSDR and other Treg-specific epigenetic signature genes including Eos, Ctla4 and Gitr, whereas CD4SP Foxp3- thymocytes analyzed as input controls were fully methylated at all epigenetic signature genes (Figure 1C and Supplementary Figure S1E) as expected from previous studies [13, 21]. Interestingly, sp-DC-induced allo-iTregs showed a weaker demethylation at TSDR, Eos, Ctla4 and Gitr, suggesting that only thymic APCs can initiate a pronounced demethylation of Treg-specific epigenetic signature genes in allo-iTregs, with t-DCs being the most efficient APC. Together, these data imply that thymic APCs not only have a preferential ability to induce Foxp3 expression, but also to provoke the epigenetic remodeling during thymic Treg development.

\section{Transcriptional profiling of mTECs, t-DCs and sp-DCs identifies various differentially expressed genes}

To unravel the unique features of thymic APCs in driving differentiation of a stable Treg lineage, we
A

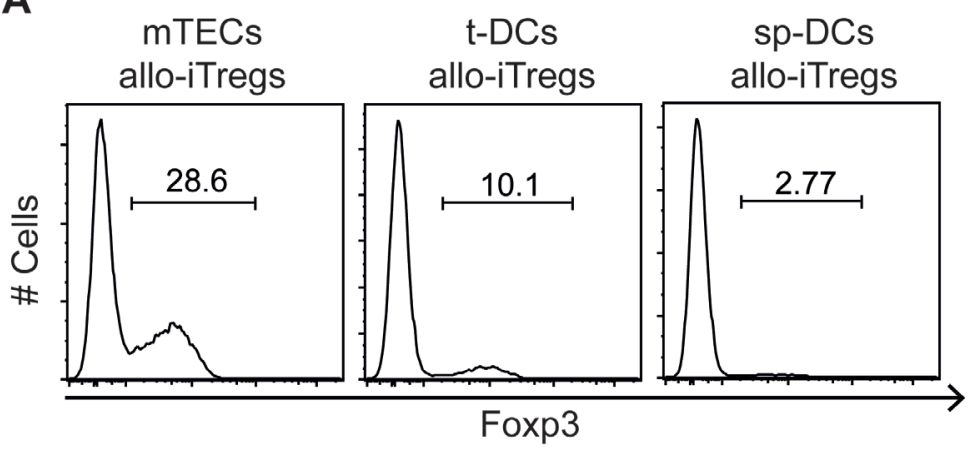

C

input

Foxp3-

mTECs

allo-iTregs

t-DCs

allo-iTregs

$\mathrm{sp}-\mathrm{DCs}$

allo-iTregs
TSDR
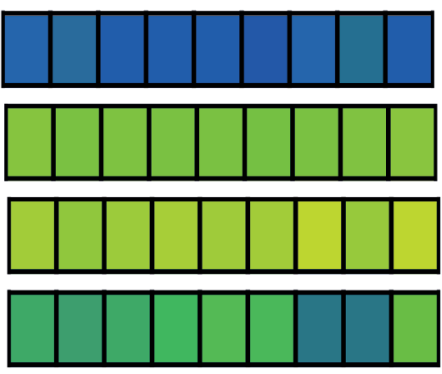

Eos
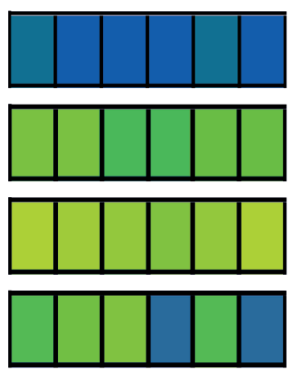

B

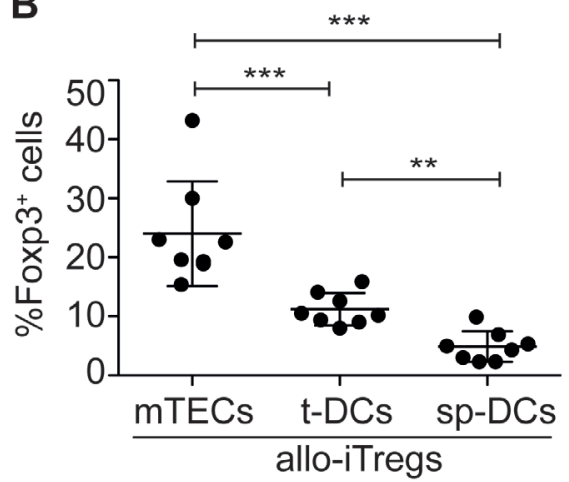

Ctla4

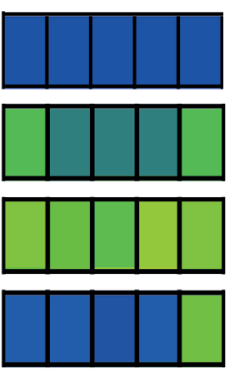

Gitr

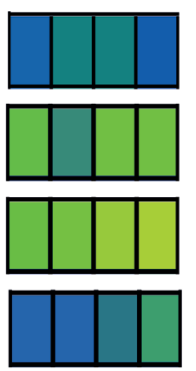

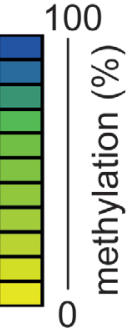

Figure 1: Thymic APCs very efficiently induce allo-iTregs with highest demethylation at TSDR and other Treg-specific epigenetic signature genes. Indicated APCs isolated from BALB/c mice were cultured with allogeneic CD4SP Foxp3 ${ }^{\text {RFP- }}$ thymocytes isolated from $\operatorname{Foxp}^{R F P} \times I L-10^{G F P}$ double reporter mice (C57BL/6 background) in presence of IL-2 for six days. A. Expression of Foxp3 in cultured $\mathrm{CD}^{+} \mathrm{CD} 90^{+}$thymocytes was analyzed by flow cytometry. Numbers indicate frequency of Foxp $3^{+}$cells. Representative data from one out of eight independent experiments are shown. B. Graph shows frequency of Foxp $3^{+}$allo-iTregs from cultures with indicated APCs. Data are summarized from eight independent experiments (mean $\pm \mathrm{SD}$ ) and tested for significance using Mann-Whitney test; $* * p$ $<0.01 ; * * * p 0.001$. C. At day 6 , Foxp $3^{\mathrm{RFP}+}$ allo-iTregs were sorted from indicated cultures, and genomic DNA isolated from these cells was analyzed for the methylation status of TSDR, Eos, Ctla4 and Gitr. Representative data from one out of six (TSDR), two (Gitr) or three (Eos and Ctla4) independent experiments are depicted. Genomic DNA from CD4SP Foxp3- thymocytes was analyzed as input control for the methylation status of TSDR (three independent samples) and Eos, Ctla4 and Gitr (unicate). Each bar represents one CpG motif. The degree of methylation at each $\mathrm{CpG}$ motif is represented according to the color code. 
performed a transcriptional profiling by RNA-Seq. To this end, mTECs and t-DCs were isolated ex vivo to high purity and sp-DCs were taken as controls (Supplementary Figure S1A). To validate the specificity of the RNA-Seq analysis, a set of previously defined control genes for mTECs and DCs was analyzed in detail. As expected, mTECs showed a higher expression of Aire, Ins 2, Foxn1 and Epcam when compared to both t-DCs and sp-DCs, which in turn showed a higher expression of Cd8a, Flt3, Itgax and Sirpa (Figure 2A). Global inspection of the RNA-Seq data by principal component analysis (PCA) and clustering of Euclidian sample distances revealed homogeneous and clearly separated clusters of sample replicates and showed a higher similarity between the two DC populations as compared to mTECs (Supplementary Figure S2). Hierarchical clustering of all differentially expressed genes (DEGs) amongst the analyzed APCs corroborated this finding (Figure 2B). Accordingly, the largest number of DEGs was found in the comparisons of mTECs with t-DCs (1555 up- and 8800 down-regulated in t-DCs) or mTECs with sp-DCs (1719 up- and 9046 down-regulated in sp-DCs) (Supplementary Table S1). Although the number of DEGs was substantially smaller when comparing sp-DCs with t-DCs (3131 up- and 1181 down-regulated in t-DCs), yet a high degree of differential gene expression could be observed. The results from these pairwise comparisons were fuelled into a KEGG pathway and GO enrichment analysis to identify functional pathways that contribute to the unique properties of thymic APCs in fostering the generation of stable Foxp $3^{+}$Tregs. Among the numerous overrepresented KEGG pathways and GO categories we focussed on those involved in cellular communication like cytokine-cytokine receptor interaction (KEGG ID: 4060), cell adhesion (KEGG ID: 4514; GO ID: 5925, 7155) and plasma membrane location (GO ID: 5886, 5887, 7166, 9897, 9986, 16020,
16021, 16324) (Supplementary Tables S2, S3). From these pathways and categories, immunologically relevant molecules were extracted and grouped into either cytokines/cytokine receptors and chemokines/chemokine receptors (Supplementary Figure S3) or cell surface/ cell adhesion-associated molecules and costimulatory molecules (Supplementary Figure S4). Detailed inspection of these extracted RNA-Seq data revealed that many genes were expressed at similar levels in both t-DCs and sp-DCs (e.g. Il1b, Il21r, Il10ra, Xcrl), confirming the more pronounced transcriptional variation between mTECs and DCs. However, t-DCs and sp-DCs still presented differential expression of a substantial number of immunologically relevant genes, and some genes, particularly those belonging to the group of costimulatory molecules (Supplementary Figure S4B), even displayed higher expression levels in both thymic APC subsets as compared to sp-DCs, making them interesting candidates contributing to the unique features of thymic APCs in driving differentiation of a stable Treg lineage.

\section{Neither costimulatory signals nor secreted factors contribute to efficient induction of allogeneic Foxp3 $^{+}$Tregs by thymic APCs}

To identify molecular factors within thymic APCs that could contribute to the engraving of specific epigenetic signatures in developing Tregs, we first focused on the differentially expressed costimulatory molecules. Flow cytometric analysis of ex vivo isolated APCs revealed a substantial expression of CD86 on all APCs, with slightly higher levels found on t-DCs (Figure 3). Interestingly, CD70, CD83, CD137L (Tnfsf9) and OX40L (Tnfsf4) were expressed at higher levels on thymic APCs when compared to sp-DCs, with again t-DCs presenting highest levels.
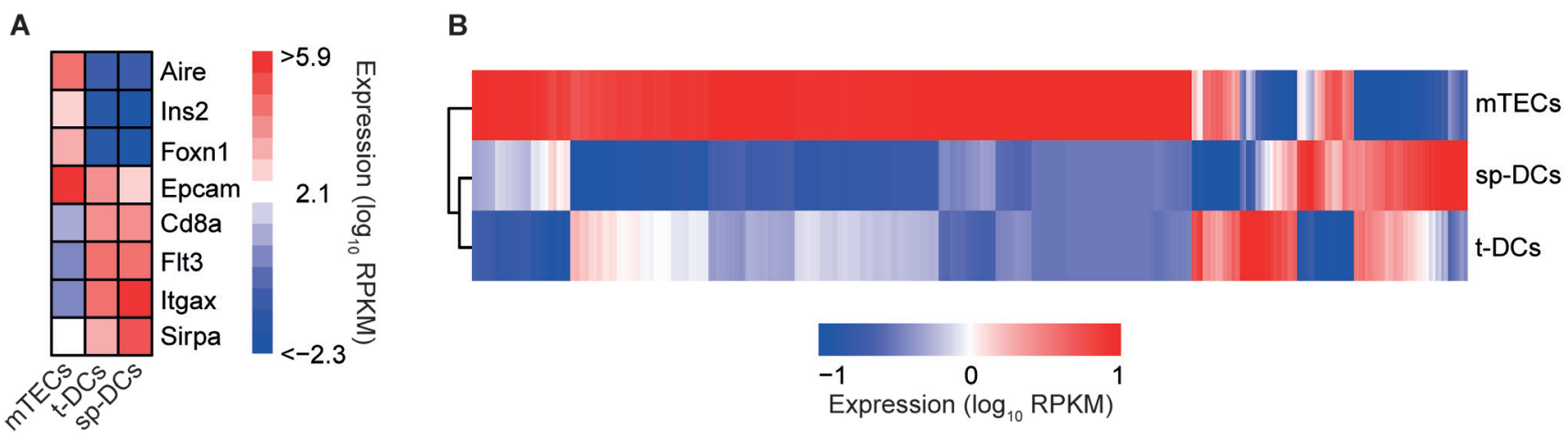

Figure 2: Global view on transcriptomes of thymic APCs. Ex vivo isolated mTECs, t-DCs and sp-DCs (all from BALB/c mice) were transcriptionally profiled by RNA-Seq. A. Heatmap analysis for expression of cell-type specific signature genes for $\log _{10}$ transformed data. Bars are color-coded according to the expression value RPKM as indicated in expression scale. Each bar is an average of three independent experiments. B. Hierarchical clustering of mean averaged over replicates and scaled gene expression values (RPKM) of all profiled genes with $D E S e q 2$ estimated $\left|\log _{2} \mathrm{FC}\right|$ of at least 1.5 and adjusted $p$-value of at most 0.05 in at least one of the performed comparisons. Bars are color-coded according to the expression value RPKM as indicated in the expression scale. Each bar is an average of three independent experiments. 
Similarly, high CD40 expression was observed for t-DC and a subset of mTECs, while sp-DCs expressed only low levels of CD40. CD40-CD40L signaling had been reported previously to influence thymic Treg development [31], however the specific contribution of CD40 expression on DCs for the in vivo generation of thymic Tregs had not been addressed so far. Hence, we here generated DCspecific CD40 knockout mice by crossing conditional CD40 knockout mice (N.G. and E. L., unpublished) to CD $11 c^{\text {Cre }}$ mice. In these mice $\left(\mathrm{CD} 11 \mathrm{c}^{\mathrm{Cre}} \mathrm{xCD} 40^{\mathrm{f} / \mathrm{fl}}\right)$, we observed a mild, but significantly reduced frequency of Foxp $3^{+}$Tregs both in thymus and spleen when compared to $\mathrm{CD} 11 \mathrm{c}^{\mathrm{WT}} \mathrm{xCD} 40^{\mathrm{f} / \mathrm{fl}}$ mice taken as controls (Figure $4 \mathrm{~A}$ ), proving that CD40 expression on DCs is critical for the in vivo generation and/or homeostasis of thymic Foxp $3^{+}$Tregs. To study the contribution of CD40-CD40L signaling to the efficient induction of allo-iTregs by thymic APCs, we next performed in vitro alloantigen-specific Treg induction cultures using mTECs, t-DCs and sp-DCs, and tested the impact of antibodies blocking CD40-CD40L signaling on the frequency of Foxp $3^{+}$allo-iTregs. For all conditions tested, no significant differences in the frequency of Foxp $3^{+}$allo-iTregs were observed upon addition of anti-CD40 as compared to isotype control antibodies (Figure 4B+4C). Importantly, interference with CD40-CD40L signaling also had no impact on the TSDR methylation status in Foxp3 $3^{+}$allo-iTregs sorted from the corresponding cultures (Figure 4D). Although these findings suggest that CD40-CD40L signaling is not critically required for the efficient induction of stable Foxp $^{+}$allo-iTregs by thymic APCs, it cannot be ruled out that mTECs and particularly t-DCs expressing high levels of CD40 had been conditioned in vivo upon receipt of signals via CD40 after encounter with $\mathrm{CD}_{40 \mathrm{~L}^{+}}$cells [39]. To study the importance of thymic APC licensing via CD40, we first temporally abrogated CD40-CD40L signaling in vivo by injecting blocking anti-CD40L into Foxp $3^{\text {GFPCre ROSA2 }} 6^{\text {RFP }}$ fate-mapping mice [40] for a period of nine days. However, this treatment did not result in any increase in exFoxp $3^{+}$cells (Supplementary Figure $\mathrm{S} 5 \mathrm{~A}, \mathrm{~S} 5 \mathrm{~B})$. Next, we performed in vitro alloantigenspecific Treg induction cultures using t-DCs from the aforementioned DC-specific CD40 knockout mice $\left(\mathrm{CD} 11 \mathrm{c}^{\mathrm{Cre}} \mathrm{xCD} 40^{\mathrm{f} / \mathrm{ft}}\right)$ as these DCs are deprived of any in vivo conditioning via CD40. Interestingly, we could not observe any difference in the frequency of Foxp $3^{+}$
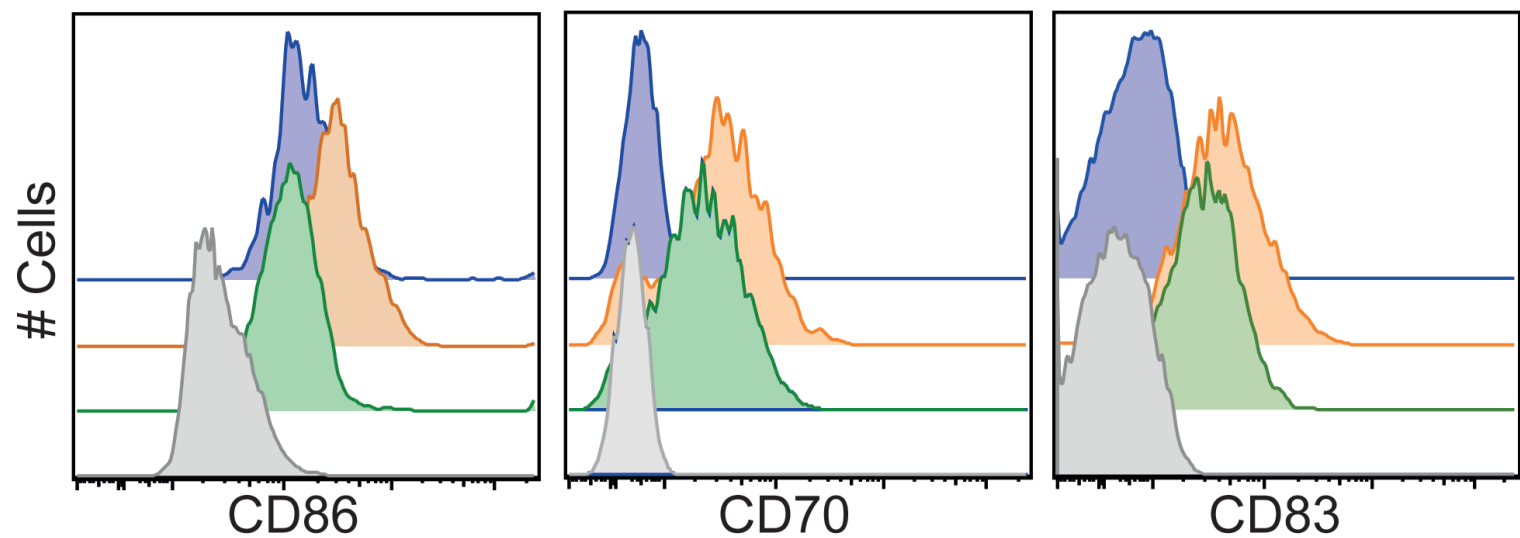

sp-DCs t-DCs MTECS control
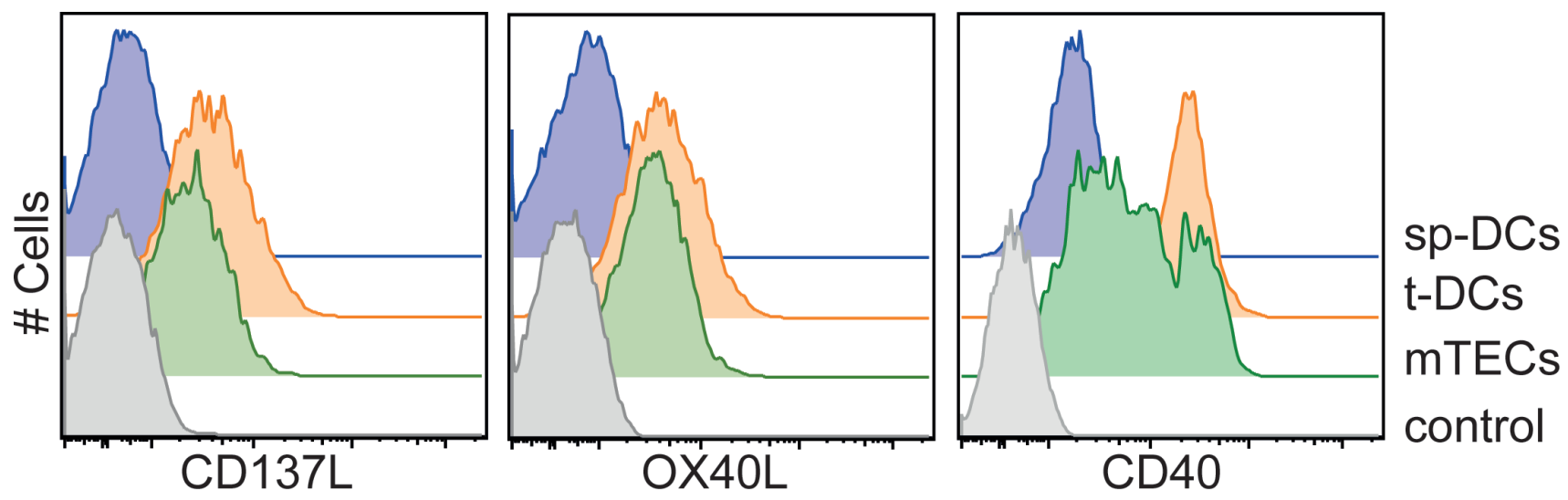

Figure 3: Expression of costimulatory molecules by thymic APCs. APCs were enriched from thymi and spleens of BALB/c mice by enzymatic digestion and gradient centrifugation. Expression of CD86, CD70, CD83, CD137L, OX40L and CD40 was analyzed on gated CD45-EpCAM+Ly51- mTECs, CD45 CD11 chi Lin $^{-}$t-DCs and CD11 $\mathrm{c}^{\text {hi }}$ Lin $^{-}$sp-DCs (Lin defined as CD90, CD49b, F4/80 and CD19) by flow cytometry. Gated CD4SP-Foxp3- thymocytes were taken as control. Representative histograms from one out of four (CD86 and CD70), five (CD40) or three (CD83, CD137L and OX40L) independent experiments are depicted. 
allo-iTregs between cultures using CD40-deficient and -proficient t-DCs (Supplementary Figure S5C), and preliminary results indicate a comparable demethylation at TSDR in Foxp $3^{+}$allo-iTregs sorted from the corresponding cultures (data not shown). Together, these findings indicate that licensing of thymic APCs via CD40 is not essential for the acquisition of their preferential ability to efficiently induce and stabilize Foxp3 expression in developing thymic Tregs.

In a next step, we investigated the functional role of other costimulatory molecules that were differentially expressed on mTECs and t-DCs compared to sp-DCs by performing in vitro alloantigen-specific Treg induction cultures in presence of blocking antibodies. Neither addition of anti-CD70, anti-CD137L and anti-OX40L alone or combined blockade of various costimulatory molecules (CD40, CD70, CD137L and OX40L) resulted in a decreased frequency of Foxp $3^{+}$allo-iTregs when compared to control cultures (Supplementary Figure S6A). Furthermore, when alloantigen-specific Treg induction cultures were performed up with APCs isolated from either CD83-mutant or CD83-transgenic mice, no differences in the frequency of Foxp $3^{+}$allo-iTregs compared to cultures with APCs isolated from WT control mice were observed (Supplementary Figure S6B). Finally, we investigated the role of soluble factors secreted by thymic APCs. For this purpose, we generated conditioned media by culturing ex vivo isolated mTECs, t-DCs and sp-DCs together with allogeneic CD4SP Foxp3- thymocytes for 48h. Addition of these conditioned media to 'fresh' alloantigen-specific Treg induction cultures did not significantly affect the frequency of Foxp $3^{+}$Tregs induced in any of the cultures (Supplementary Figure S6C). Together, these data ruled out any significant contribution of major costimulatory
A

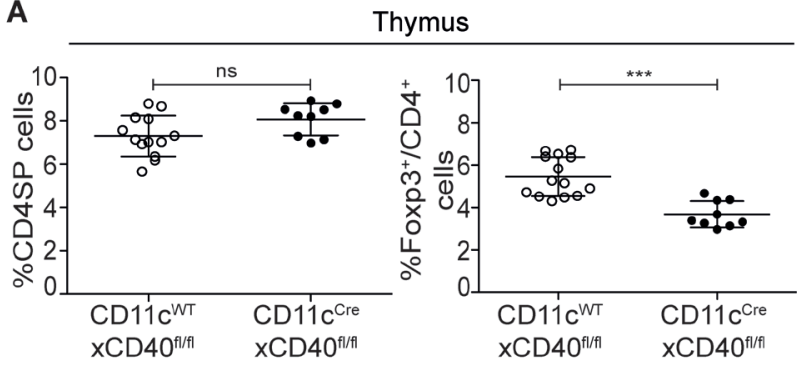

B

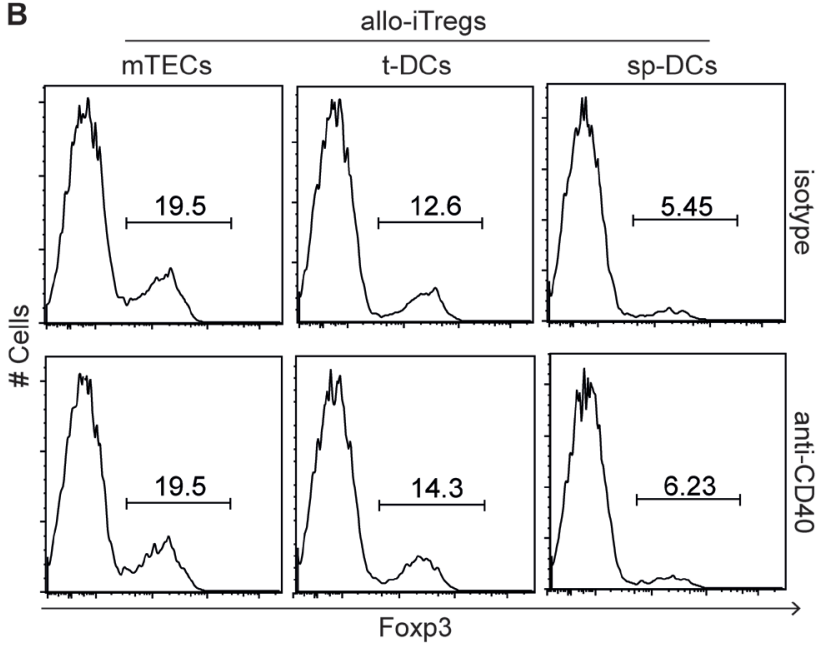

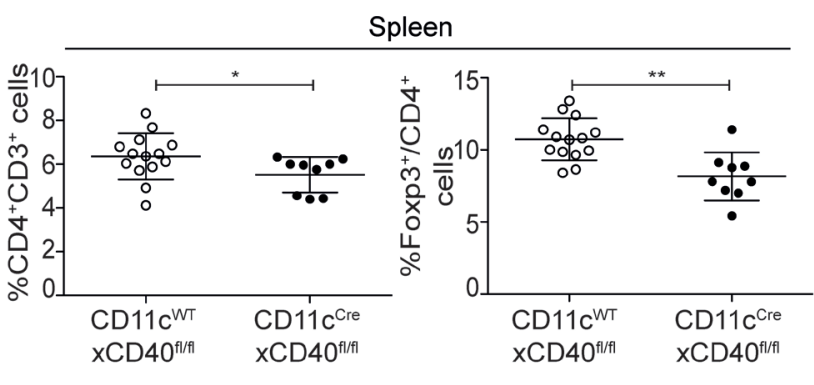

C

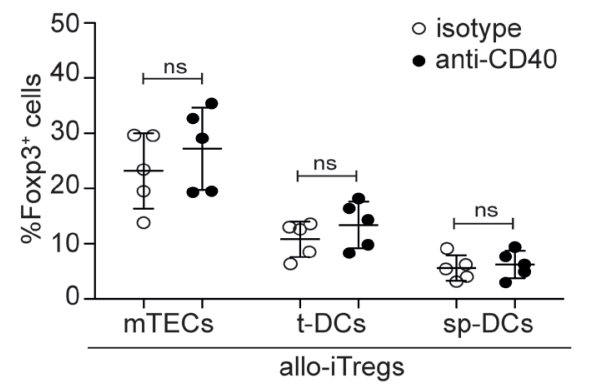

D

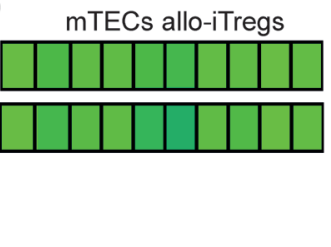

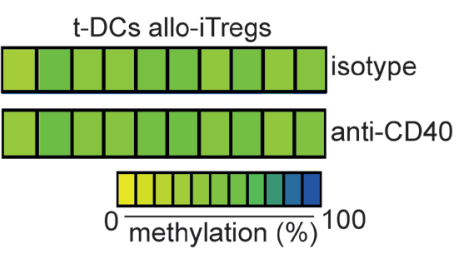

Figure 4: CD40-CD40L signaling is not critically required for generation of stable allo-iTregs. A. Thymocytes and splenocytes from CD11 $\mathrm{c}^{\mathrm{Cre}} \mathrm{xCD} 40^{\mathrm{f} / \mathrm{fl}}$ (DC-specific CD40 knockout, filled circles) and CD11 $\mathrm{c}^{\mathrm{WT}} \mathrm{xCD} 40^{\mathrm{f} / \mathrm{fl}}$ mice $(\mathrm{CD} 40$ competent control, open circles) were analyzed by flow cytometry. Graphs show frequency of CD4SP thymocytes, Foxp $3^{+}$cells among CD4SP thymocytes, $\mathrm{CD}^{+} \mathrm{CD}^{+}$splenocytes and Foxp3 $3^{+}$Tregs cells among $\mathrm{CD}^{+} \mathrm{CD}^{+}$splenocytes. Data are summarized from two independent experiments (mean $\pm \mathrm{SD}$ ) and tested for significance using Mann-Whitney test; ${ }^{*} p<0.05 ; * *<0.01 ; * * *<0.001$; ns, not significant. (B-D) Indicated APCs (BALB/c) were cultured with CD4SP-Foxp3 ${ }^{\text {RFP- }}$ thymocytes $(\mathrm{C} 57 \mathrm{BL} / 6)$ in presence of IL-2 and either anti-CD40 or isotype control antibodies for six days. B. Expression of Foxp3 in cultured $\mathrm{CD} 4{ }^{+} \mathrm{CD} 90^{+}$thymocytes was analyzed by flow cytometry. Numbers indicate frequency of Foxp $3^{+}$cells. Representative data from one out of five independent experiments are depicted. C. Graph shows frequency of Foxp $3^{+}$allo-iTregs from cultures with indicated APCs in presence of anti-CD40 (filled circles) or isotype control antibodies (open circles). Data are summarized from five independent experiments (mean \pm SD) and tested for significance using Mann-Whitney test; ns, not significant. D. At day 6, Foxp3 ${ }^{\text {RFP+ }}$ allo-iTregs were sorted from indicated cultures, and genomic DNA isolated from these cells was analyzed for the methylation status of TSDR. Representative data from one out of three independent experiments are depicted. Each bar represents one $\mathrm{CpG}$ motif. The degree of methylation at each $\mathrm{CpG}$ motif is represented according to the color code. 
molecules expressed on thymic APCs and secretory factors released by them, in the generation of stable alloantigenspecific Foxp $3^{+}$Tregs.

\section{Phenotype and in vitro suppressive properties of thymic APC-induced allo-iTregs}

The interesting finding of thymic APCs being prime cellular players in initiating the demethylation at
Treg-specific epigenetic signature genes in allo-iTregs, prompted us to phenotypically and functionally characterize these allo-iTregs. First, expression of IL-10 and IFN- $\gamma$, two cytokines known to play an important role for the suppressive activity of Foxp $3^{+}$Tregs [41], was analyzed. While mTEC-induced allo-iTregs completely lacked IL-10 expression, a significant fraction of t-DCinduced allo-iTregs expressed IL-10, comparable to sp-DC-induced allo-iTregs (Figure 5A and Supplementary Figure S7A). On the contrary, mTEC-induced allo-iTregs
A
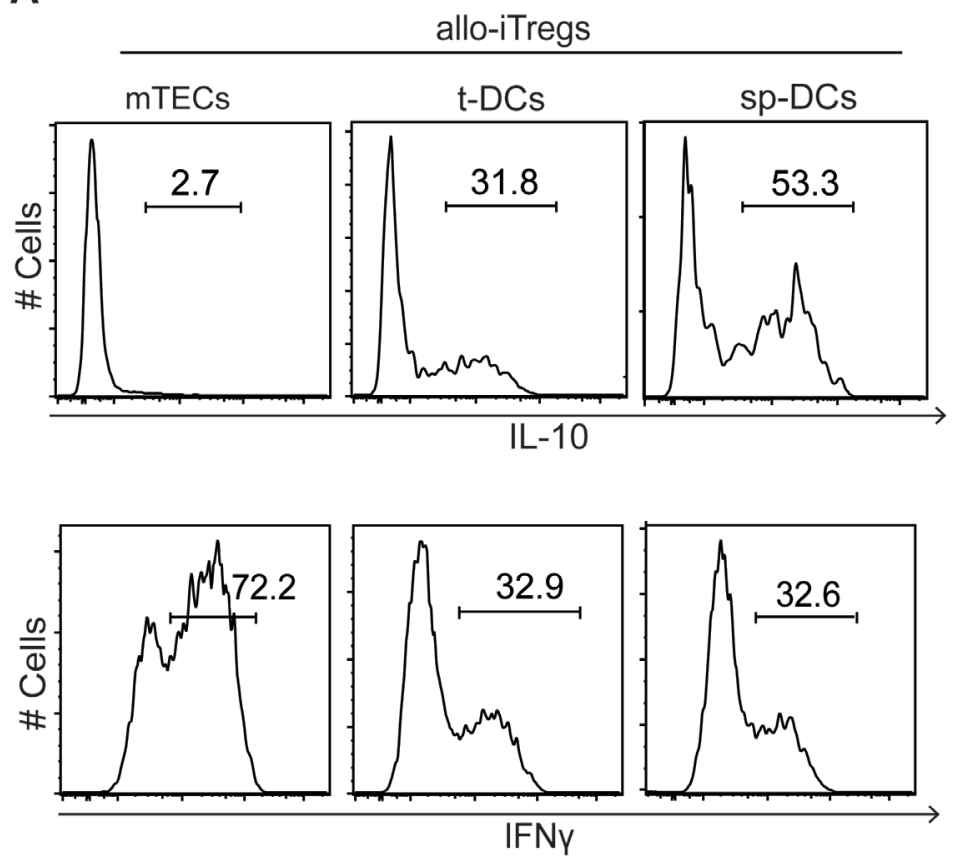

B
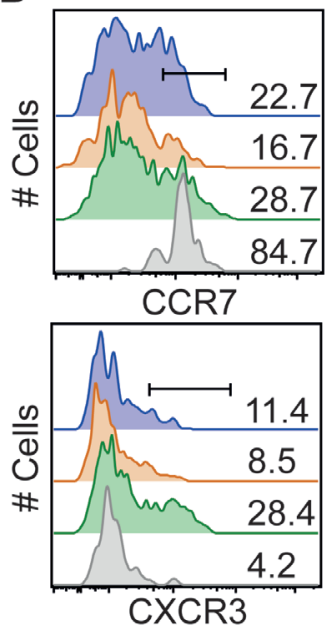

Foxp3 ex vivo t-DCs allo-iTregs mTECs allo-iTregs sp-DCs allo-iTregs

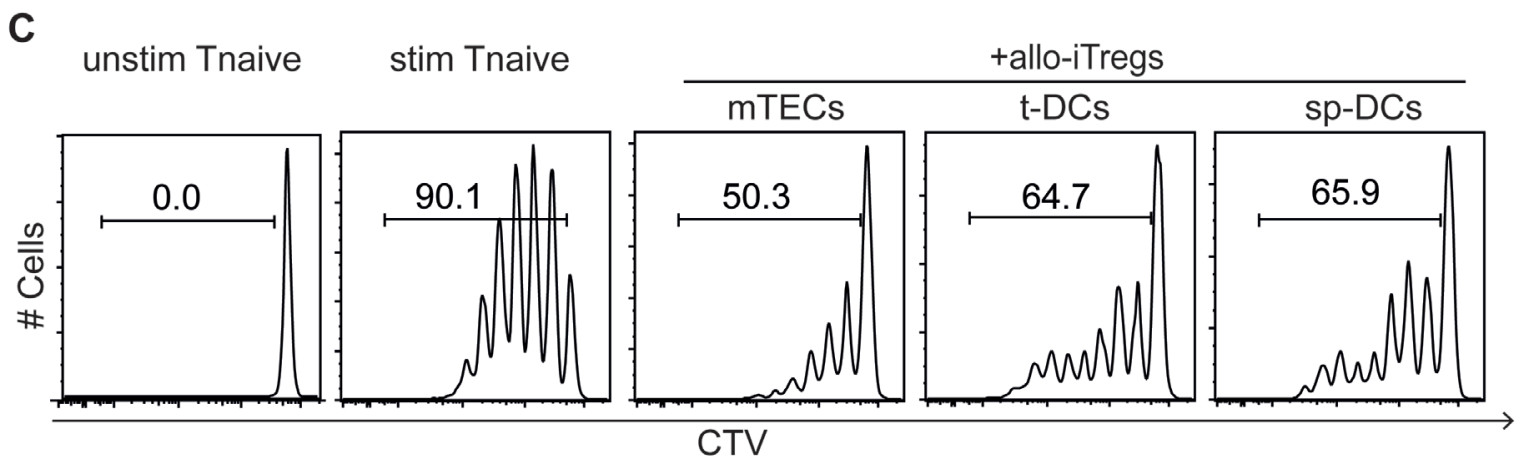

Figure 5: In vitro phenotypic and functional characterization of allo-iTregs generated by thymic APCs. Indicated APCs $(\mathrm{BALB} / \mathrm{c})$ were cultured with CD4SP Foxp3 ${ }^{\text {RFP- }}$ thymocytes (C57BL/6) in presence of IL-2 for six days. A. At day 6, cells were restimulated with phorbol 12-myristate 13-acetate and ionomycin for detection of IFN- $\gamma$, and expression of IL-10 ${ }^{\mathrm{GFP}}$ (top) and IFN- $\gamma$ (bottom) was assessed by flow cytometry in gated Foxp $3^{\mathrm{RFP}+}$ allo-iTregs from indicated cultures. Numbers indicate frequency of IL- $10^{+}$and IFN- $\gamma^{+}$ cells. Representative data from one out of four independent experiments are depicted. B. At day 6, expression of CCR7 (top) and CXCR3 (bottom) was directly assessed by flow cytometry on gated Foxp3 ${ }^{\text {RFP+ }}$ allo-iTregs from indicated cultures. Ex vivo isolated CD4SP Foxp3thymocytes served as controls. Numbers indicate frequency of CCR $7^{+}$and CXCR $3^{+}$cells. Data were taken from one out of two independent experiments. C. At day 6, Foxp3 ${ }^{\text {RFP+ }}$ allo-iTregs were sorted from indicated cultures by flow cytometry. Freshly isolated, CTV-labeled naïve $\mathrm{CD}^{+} \mathrm{T}$ cells were stimulated with anti-CD3/anti-CD28 beads in presence of indicated allo-iTregs at a ratio of 1:4 (Tregs to naïve T cells). Naïve T cells stimulated in absence of allo-iTregs (stim Tnaive) as well as naïve T cells receiving no stimulus (unstim Tnaive) served as controls. After four days, proliferation of naïve T cells was assessed by measuring CTV dilution in living naïve $\mathrm{CD} 4^{+} \mathrm{CD} 90.2^{+} \mathrm{CD} 45.1^{+}$ $\mathrm{T}$ cells by flow cytometry. Numbers indicate frequency of cells in indicated gates. Representative data from one out of four independent experiments are depicted. 
showed a significantly enhanced expression of IFN- $\gamma$ when compared to both t-DC- and sp-DC- induced allo-iTregs. Although a similar cytokine expression profile was observed in corresponding Foxp $3 \mathrm{CD}^{+} \mathrm{T}$ cells from the same alloantigen-specific cocultures (Supplementary Figure S7B, S7C), we could not detect any difference in the expression of IL-10 and IFN- $\gamma$ within ex vivo isolated Foxp $3^{+}$Tregs from thymus and spleen (Supplementary Figure S7D), suggesting that the abovementioned cytokine expression patterns were preferentially induced in the alloantigen-specific cultures. Next, expression of chemokine receptors CCR7 and CXCR3 was determined on the different allo-iTregs to assess their recirculating and inflammation-seeking capacity, respectively [42]. No differences in CCR7 expression were observed between mTEC-, t-DC- and sp-DC-induced allo-iTregs, while mTEC-induced allo-iTregs showed a higher frequency of $\mathrm{CXCR}^{+}$cells when compared to both DC-induced
allo-iTregs (Figure 5B and Supplementary Figure S7E). Finally, we tested mTEC-, t-DC- and sp-DC-induced allo-iTregs in an in vitro suppression assay and observed a comparable suppressive capacity for all allo-iTregs (Figure 5C and Supplementary Figure S7F), suggesting that the differences in the methylation status at Tregspecific epigenetic signature genes do not affect in vitro suppressive function.

\section{Thymic APC-induced allo-iTregs delay skin graft rejection}

Previous studies have demonstrated that iTregs although being functional in vitro lack suppressive potential when tested in vivo, a finding that was explained by the fully methylated Treg-specific epigenetic signature genes and their instable phenotype $[11,13]$. Thus, we
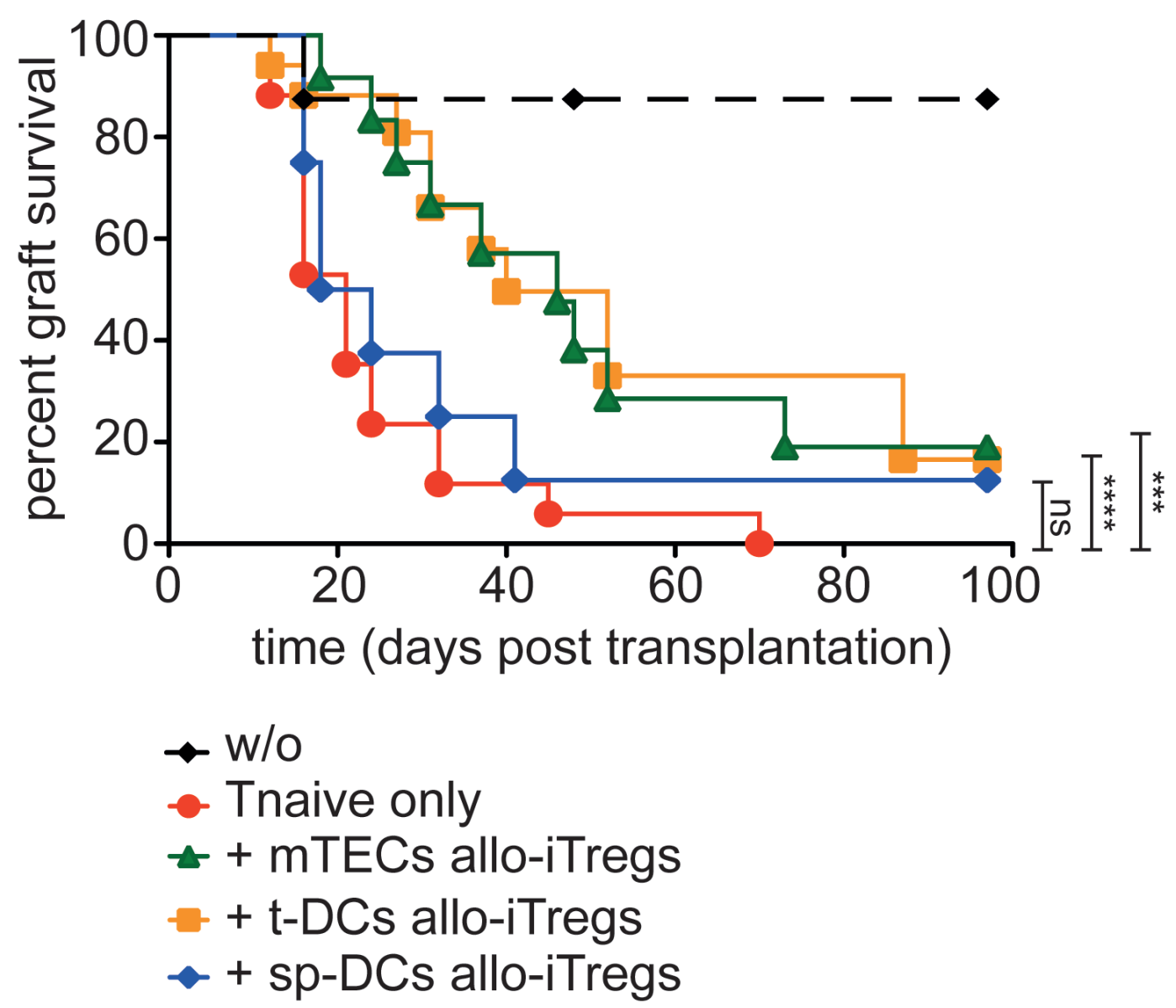

Figure 6: Allo-iTregs generated by thymic APCs very efficiently suppress skin allograft rejection. Indicated APCs $(\mathrm{BALB} / \mathrm{c})$ were cultured with CD4SP Foxp3 ${ }^{\text {RFP- }}$ thymocytes $(\mathrm{C} 57 \mathrm{BL} / 6)$ in presence of IL-2 for six days. One day prior to transplantation, allo-iTregs from indicated cultures were sorted by flow cytometry and injected into Rag $2^{-/}$mice. Mice were additionally injected with $\mathrm{CD} 4^{+} \mathrm{CD} 90.2^{+} \mathrm{CD} 45.1^{+} \mathrm{CD} 25^{-} \mathrm{CD} 44^{-} \mathrm{CD} 62 \mathrm{~L}^{\text {hi }}$ naïve $\mathrm{T}$ cells. Mice receiving no cells (w/o) or naïve T cells only served as controls. One day later, allogeneic skin transplantation was performed. The following days animals were monitored for signs of rejection until day 100. Relative graft survival for each group is depicted and data are cumulative of three independent experiments; w/o ( $n=6$, black diamond), Tnaive only $(n=13$, red circle $),+$ mTEC allo-iTregs $(n=11$, green triangle $),+$ t-DC allo-iTregs $(n=12$, orange square $)$ and + sp-DC allo-iTregs $(n=8$, blue diamond). Significance of survival graph was calculated between mice receiving Tnaive only versus mice cotransferred with different APC-induced allo-iTregs using log-rank (mantel cox) test. ${ }^{* * *} p<0.001 ; * * * * p<0.0001$; ns, not significant. 
here tested the in vivo suppressive capacity of thymic APC-induced allo-iTregs, which show a pronounced demethylation at the TSDR and other Treg-specific epigenetic signature genes, in a highly immunogenic, allogeneic skin transplantation model. Thereto, allo-iTregs together with congenically marked (CD45.1) $\mathrm{CD}^{+}$naïve $\mathrm{T}$ cells were adoptively transferred into Rag2/- $(\mathrm{C} 57 \mathrm{BL} / 6)$ mice, one day prior to the skin transplant from BALB/c mice (Supplementary Figure S8A). Mice receiving $\mathrm{CD}^{+}$naïve $\mathrm{T}$ cells only or receiving no cells served as graft rejection or graft survival controls, respectively. Rapamycin was given to all groups on day -1, day 0 and day 2 in order to moderately suppress immediate triggering of the alloresponse. Graft survival was tracked until day 100 and the time point of graft rejection or day 100 in case of graft survival defined the end point. As expected, the group receiving only $\mathrm{CD} 4^{+}$naïve $\mathrm{T}$ cells rejected the skin graft and mice receiving no cells accepted the graft (Figure 6). Interestingly, allo-iTregs induced by both mTECs and t-DCs significantly improved graft survival in comparison to the group receiving $\mathrm{CD} 4^{+}$naïve $\mathrm{T}$ cells only. In contrast, sp-DC-induced allo-iTregs were incapable of significantly prolonging allograft survival (Figure 6), showing that only thymic APC-generated allo-iTregs display suppressive potential in vivo.

When we analyzed frequency and total cell number of adoptively transferred $\mathrm{CD}^{+}$naïve $\mathrm{T}$ cells and allo-iTregs at the individual end points within lymph nodes draining the skin transplantation site, we did not observe overt differences between the different groups (Supplementary Figure S8B-D). Furthermore, no differences could be detected regarding the frequency of de novo induced Foxp $3^{+}$Tregs among adoptively transferred $\mathrm{CD}^{+}$naïve $\mathrm{T}$ cells (Supplementary Figure $\mathrm{S} 8 \mathrm{E})$. However, we observed mild differences in the stability of Foxp3 expression among adoptively transferred allo-iTregs as both mTEC- and t-DC-induced allo-iTregs showed a slightly increased stability when compared to sp-DC-induced allo-iTregs (Supplementary Figure S8F). Taken together, these observations indicate that alloreactive Tregs induced by thymic APCs show a trend of higher maintenance of Foxp3 expression in vivo and can significantly prolong skin graft survival.

\section{DISCUSSION}

Solid organ transplantation remains the only treatment option for several end-stage organ failures of heart, lung, kidney and liver; it yet comes with a hitch, graft rejection and side effects of unspecific immunosuppression. Immune cells of the organ recipient recognize and attack the graft as foreign, due to donorrecipient mismatch of major and minor histocompatibility complex antigens. Lately, use of immunosuppressive Tregs as cellular therapy has gained increasing interest to promote long-term graft survival by induction of immune tolerance $[14,43,44]$. However, the proposed clinical trials using polyclonal Tregs require cells in excess to efficiently suppress graft rejection [45], this can in turn result in increased immunosuppression and thereby increases the risk of infections. Thus, current concepts favor the use of alloantigen-specific Foxp $3^{+}$Tregs since the higher specificity of such allo-Tregs will reduce the number of Tregs required for injection into patients to promote graft survival. Hence, an accumulating body of knowledge about the benefits of using allo-Tregs to attain transplantation tolerance makes them promising candidates for therapeutic use [2, 46-48]. On the obverse side of the coin, there are still limitations in identifying stable allo-Tregs ex vivo. In addition, lack of standard protocols for in vitro generation of stable Tregs with alloantigen-specificity is a further major drawback. This is due to transient upregulation of Foxp3 expression by conventional $\mathrm{T}$ cells upon their activation or due to downregulation of Foxp3 expression in Tregs under certain inflammatory conditions $[40,49]$. Therefore, the present study aimed at identifying cellular players and molecular mediators involved in Treg cell fate determination and maintenance, which could enable efficient in vitro generation of allo-Tregs with a stable phenotype.

We could demonstrate that allo-iTregs induced by thymic APCs show an increased efficacy in delaying skin graft rejection, whereas allo-iTregs induced by sp-DCs failed to prolong graft acceptance. Keeping feasible numbers in mind, low amounts of allo-iTregs were used in this preclinical model. Though the graft could not be fully rescued due to the high immunogenicity of the skin transplant, these numbers were enough to prolong graft acceptance by thymic APC-induced allo-iTregs. Two recent reports are supporting our finding, where one showed that $\mathrm{Nrp}^{+}$Tregs generated in the thymus could suppress skin graft rejection and another stated human thymus to be an important source of stable and suppressive Foxp $3^{+}$Tregs for clinical therapeutics $[50,51]$.

Although thymic APC-induced allo-iTregs showed the highest maintenance of Foxp3 expression upon in vivo transfer, a substantial fraction of allo-iTregs induced by sp-DCs could also retain Foxp3 expression. In accordance with our recent findings $[13,18,19]$, these data suggest that mere Foxp3 expression is not sufficient to confer full functional competence to Tregs. Epigenetic remodeling of not only the Foxp3 locus, but also of other Tregspecific epigenetic signature genes like Eos, Ctla4 and Gitr is of utmost importance for the acquisition of their full suppressive capacity. Eos, CTLA-4 and GITR have been reported to be associated with stability, functional capacity and thymic differentiation of Tregs [52-55], and demethylation of these Treg-specific genes supports maintenance of their expression, being critical to establish stable Treg functional properties. Thus, the higher efficiency of mTECs and t-DCs as compared to sp-DCs to induce an epigenetic remodeling at Eos, Ctla 4 and Gitr 
might be causative for the superior in vivo suppressive capacity of thymic APC-induced allo-iTregs. It is worth mentioning that sp-DC-induced allo-iTregs also displayed a substantial demethylation at Treg-specific epigenetic signature genes, albeit at lower levels when compared to thymic APC-induced allo-iTregs, suggesting that also sp-DCs have the ability to induce epigenetic remodeling. The induction of Foxp3 expression and the formation of a Treg-specific epigenome are two independent events [21], which depend on the strength of TCR signaling and on the duration of TCR engagement [13]. Thus, it can be hypothesized that the lower demethylation at Treg-specific genes in sp-DC-induced Tregs could be an outcome of the reduced duration of TCR engagement by sp-DCs. We also observed a higher potential of thymic APCs to induce a high frequency of allo-iTregs, further supporting the essential role of thymic APCs for the generation of organspecific Tregs within the thymus [56] and suggesting that also differences in the TCR repertoires of allo-iTregs induced by mTECs, t-DCs and sp-DCs might contribute to their in vivo functional properties [57]. Together, our findings indicate that thymic APCs express unique characteristics supporting both the induction and fate determination of Foxp $3^{+}$Tregs.

Additional to initiating the described epigenetic remodeling in allo-iTregs by thymic APCs, expression of both IL-10 and IFN- $\gamma$ by t-DC-induced allo-iTregs and primarily expression of IFN- $\gamma$ by mTEC-induced allo-iTregs was also observed. Both cytokines are associated with preventing allogeneic skin graft rejection and inhibiting GvHD progression [58-60]. Increased CXCR3 expression mainly by mTEC-induced allo-iTregs could be a result of high IFN- $\gamma$ expression by these cells, which can induce CXCR3 expression in Tregs, and can effectively suppress allogeneic graft rejection $[61,62]$. Since post-positive selection thymocytes require CCR7 for cortico-medullary migration, Foxp3thymocytes used for allo-iTreg generation in vitro were largely $\mathrm{CCR}^{+}$. The comparable expression of $\mathrm{CCR} 7$ by the different allo-iTregs is hence likely to reflect their shared developmental stage, rather than shared functional characteristics. Although we cannot exclude that IL-10, IFN- $\gamma$, CCR7 and CXCR3 play a critical role for the suppression of skin graft rejection by allo-iTregs, they cannot account for the different in vivo suppressive properties observed for t-DC- and sp-DC-induced allo-iTregs as both allo-iTregs showed largely comparable cytokine and chemokine receptor expression patterns.

Gene expression profiling of APCs revealed highest differential gene expression by mTECs in comparison to DCs, possibly due to promiscuous tissue-specific gene expression by mTECs [63]. In addition, their shared developmental origin and migration of peripheral DCs into the thymus may account for the relative similarity of t-DCs and sp-DCs transcriptional profiles [64]. Many of the DEGs could be involved in the higher potency of thymic APCs in driving differentiation of a stable Treg lineage. The in vivo analysis of DC-specific CD40 knockout mice clearly indicated that CD40 expression on DCs is critical for the in vivo generation and/or homeostasis of thymic Foxp $3^{+}$Tregs, expanding our previous knowledge on the impact of CD40-CD40L signaling for the generation of tTregs [31, 65]. However, we could provide experimental evidence that licensing of thymic APCs via CD40 is not essential for the acquisition of their preferential ability to efficiently induce and stabilize Foxp3 expression in developing thymic Tregs. Furthermore, neither CD40-CD40L signaling, signals delivered through other major costimulatory molecules on thymic APCs nor secretory factors released by them seemed to be essential for the generation of stable alloantigen-specific Foxp $3^{+}$Tregs. Thus, the molecular factors within thymic APCs that could contribute to the engraving of specific epigenetic signatures in developing Tregs remain to be identified. These findings will be instrumental in directing efficient use of in vitro generated stable Tregs in therapeutic restoration of tolerance in clinical transplantation and autoimmune diseases.

\section{MATERIALS AND METHODS}

\section{Animals}

BALB/c mice and Rag2 $2^{-/}$mice were purchased from Janvier and Jackson, respectively. Foxp $3^{R F P} \times I L$ $10^{G F P}$ double reporter mice (C57BL/6 background) [66, 67], kindly provided by Richard Flavell (Yale University School of Medicine, New Haven, USA), C57BL/6 x CD45.1 mice, Foxp $3^{\mathrm{hCD} 2}$ mice (BALB/c background) and mice with a DC-specific deletion of CD40 were bred at the animal facility of the Helmholtz Centre for Infection Research (Braunschweig, Germany). The conditional CD40 knockout mice (N.G. and E.L., unpublished) contained loxP sites upstream of exon 2 and downstream of exon 3. Cre-mediated deletion of the two, floxed' exons not only eliminates sequences encoding the first and second TNFR-type Cys-rich domains, but also induces a frameshift mutation, thereby creating a non-functional allele. To generate mice with a DC-specific CD40 deletion, $\mathrm{Cd} 40^{\mathrm{f} / \mathrm{fl}}$ mice were crossed with $\mathrm{CD} 11 \mathrm{c}^{\mathrm{Cre}}$ mice [68]. CD83 transgenic mice (CD83tg, C57BL/6 background), expressing the CD83 molecule under control of the MHCI promoter [69], and CD83 mutant mice (CD83 ${ }^{\text {lcd4}}$, C57BL/6 background), harbouring a missense mutation in the last exon of CD83 [70], were both bred at the animal facility of the Bernhard-Nocht-Institute for Tropical Medicine (Hamburg, Germany). Foxp $3^{\text {GFPCre }}$ ROSA26 ${ }^{\text {RFP }}$ fate-mapping mice [40] were bred at the animal facility of the RIKEN Center for Integrative Medical Sciences. Rag2 $^{-/}$mice were used at the age of eight weeks at the 
time of experimental procedure. All other mice were used at the age of four to ten weeks. All mice were housed and handled under specific pathogen-free conditions under supervision of institutional animal welfare officers and in accordance with approved protocols from the Institutional Animal Care at RIKEN or good animal practice as defined by FELASA and the national animal welfare body GV-SOLAS. The animal protocol was approved by the Niedersächsisches Landesamt für Verbraucherschutz und Lebensmittelsicherheit: animal licensing committee permission no. 33.14-42502-04-10/0071. Animal experiments were performed in accordance with institutional, state, and federal guidelines. Animals were handled with appropriate care and welfare, and all efforts were made to minimize suffering.

\section{Antibodies and flow cytometry}

Cell suspensions from lymphoid organs were stained with fluorochrome-conjugated anti-mouse CD3 (17A2), CD4 (RM4-5), CD8a (53-6.7), CD11c (N418), CD19 (6D5), CD25 (PC61.5), CD40 (1C10), CD44 (IM7), CD45 (30-F11), CD45.1 (A20), CD49b (DX5), CD62L (MEL14), CD70 (FR70), CD83 (Michel-17), CD86 (GL1), CCR7 (4B12), CXCR3 (CXCR3-173), EpCAM (G8.8), F4/80 (BM8), Ly51 (6C3), Ly51 (FG35.4), CD90.1 (HIS51), CD90.2 (53-2.1), CD137L (TKS-1), CD252 (RM134L), TCR $\beta$ (H57-597) and anti-human CD2 (RPA2.10) that were purchased from either BD Biosciences, Biolegend or eBioscience. Intracellular staining was performed with Foxp3 staining kit for Foxp3 (FJK-16s) and IFN- $\gamma$ (XMG1.2) Abs according to manufacturer's instructions (eBioscience). For IFN- $\gamma$ cytokine staining, cells were stimulated with phorbol 12-myristate 13-acetate (Sigma) and ionomycin (Sigma) for $4 \mathrm{~h}$ and with brefeldin A for the last 2 h. For dead cell exclusion LIVE/DEAD ${ }^{\circledR}$ fixable Near-IR stain kit (Invitrogen) or Sytox Blue (Molecular Probes) was used. Flow cytometric analysis was performed on LSR II (BD Biosciences) and data were analyzed using FlowJo software (Tree Star).

\section{Purification of thymocytes and peripheral $T$ cells}

For purification of CD4SP thymocytes from male or female donor mice, total thymocytes were depleted of $\mathrm{CD}^{+}$cells using APC-conjugated anti-CD8 ${ }^{+}$and antiAPC microbeads (Miltenyi Biotec) followed by magnetic separation using the autoMACS separation system (Miltenyi Biotec). Peripheral CD4 ${ }^{+} \mathrm{T}$ cells were enriched from pooled spleen and lymph node cells using anti-CD4 microbeads followed by magnetic separation using the autoMACS separation system. CD4SP Foxp3- thymocytes or $\mathrm{CD}^{+}{ }^{+} \mathrm{Foxp}^{-}$peripheral T cells were sorted as $\mathrm{CD}^{+}{ }^{+} \mathrm{RFP}-$

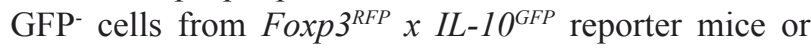
stained with anti-human CD2 to isolate CD4SP Foxp3 $3^{\mathrm{hCD} 2-}$ thymocytes from Foxp $3^{\mathrm{hCD} 2}$ mice on FACS Aria II (BD Biosciences). For the in vitro and in vivo suppression assays, naïve $\mathrm{CD}^{+} \mathrm{T}$ cells were isolated from pooled spleen and lymph node cells from CD45.1 congenic C57BL/6 mice. Briefly, CD4 ${ }^{+} \mathrm{T}$ cells were enriched using anti-CD4 microbeads followed by magnetic separation using the autoMACS separation system. Subsequently, naïve $\mathrm{CD}^{+} \mathrm{T}$ cells $\left(\mathrm{CD} 4^{+} \mathrm{CD} 90.2^{+} \mathrm{CD} 25^{-} \mathrm{CD} 44^{-} \mathrm{CD}^{-} \mathrm{L}^{\mathrm{hi}}\right.$ ) were sorted on FACS Aria II.

\section{Purification of APCs}

To isolate thymic APCs and sp-DCs, thymic lobes and spleens were separated from male or female mice, finely chopped into pieces and digested in complete RPMI 1640 medium (Life Technologies) containing 0.2 $\mathrm{mg} / \mathrm{ml}$ collagenase/dispase (Roche), $0.25 \mathrm{mg} / \mathrm{ml}$ DNase $\mathrm{I}$ (Roche) and incubated at $37^{\circ} \mathrm{C}$ for $1 \mathrm{~h}$. Liberated cells were filtered through $100 \mu \mathrm{M}$ nylon mesh and subjected to percoll gradient using $1.115 \mathrm{~g} / \mathrm{ml}$ high-density percoll and $1.06 \mathrm{~g} / \mathrm{ml}$ of low-density percoll. The gradient was centrifuged at $1350 \mathrm{~g}$ for $30 \mathrm{~min}$ at $4^{\circ} \mathrm{C}$. The low-density interface was collected for APCs. Cells were sorted as CD45-EpCAM ${ }^{+}$Ly51- for mTECs, CD $45^{+} \mathrm{CD} 11 \mathrm{c}^{\text {hi }}$ Lin $^{-}(\mathrm{Lin}$ is defined as CD90, CD49b, F4/80 and CD19) for t-DCs and CD11 $\mathrm{c}^{\text {hi }}$ Lin $^{-}$for sp-DCs on FACS Aria II.

\section{Cell culture}

Cells were cultured in RPMI 1640 medium (Life Technologies) supplemented with penicillin $(50 \mathrm{U} /$ $\mathrm{ml})$, streptomycin (50 U/ml), HEPES (25 mM), sodium pyruvate (1 $\mathrm{mM}), \beta$-mercaptoethanol $(50 \mu \mathrm{M})$ (all purchased from Biochrom) containing $10 \%$ fetal calf serum (Sigma-Aldrich), at $37^{\circ} \mathrm{C}, 5 \% \mathrm{CO}_{2}$ in $96 \mathrm{U}$ bottom plates (Corning).

\section{In vivo blocking of CD40-CD40L signaling}

Male or female Foxp3 $3^{\text {GFPreROSA26 }} 6^{\text {RFP }}$ fatemapping mice were repetitively injected i.p. with 0.3 $\mathrm{mg}$ anti-CD40L (MR1) in $300 \mu \mathrm{l}$ PBS at days 0, 2, 4, 6 and 8. Control mice received PBS injections. At day 9, mice were sacrificed and the frequency of total Foxp $3^{\mathrm{GFP}+}$ as well as exFoxp3 $3^{+}$cells $\left(\mathrm{GFP}^{-} \mathrm{RFP}^{+}\right)$among CD4SP thymocytes and splenic $\mathrm{CD}^{+} \mathrm{T}$ cells was determined by flow cytometry.

\section{In vitro Treg differentiation assay}

For the alloantigen-specific system, 10x10 $\mathrm{CD}^{+}{ }^{+}$Foxp $^{-}$cells derived from $F$ oxp $3^{R F P} \times I L-10^{G F P}$ double reporter mice (C57BL/6 background) or Foxp $3^{\mathrm{hCD} 2}$ (BALB/c background) were plated with either mTECs 
(APC to $\mathrm{T}$ cell ratio 1:50 if not indicated otherwise) or DCs (APC to $\mathrm{T}$ cell ratio 1:10 if not indicated otherwise) in presence of $100 \mathrm{ng} / \mathrm{ml}$ recombinant mouse IL-2 (R\&D systems) for six days. For in vitro blocking experiments, titrated amounts of anti-CD40 (HM40-3, Biolegend), antiCD70 (FR70, Biolegend), anti-CD137 (TKS-1, Biolegend) and anti-OX40L (RM134L, Biolegend) was used. At the end of the cultures, allo-iTregs were either directly phenotypically analyzed or sorted as $\mathrm{CD} 4^{+} \mathrm{CD} 90^{+} \mathrm{Foxp} 3^{+}$ cells for subsequent analyses on FACS Aria II using the Foxp3 reporter molecules RFP or hCD2.

\section{In vitro Treg suppression assay}

Sorted allo-iTregs were cultured with freshly isolated, Cell Trace Violet (CTV) ${ }^{\mathrm{TM}}$ (Invitrogen)-labeled naïve $\mathrm{CD}^{+} \mathrm{T}$ cells at ratios from 1:4 to 1:32 (Tregs to naïve $\mathrm{T}$ cells). Anti-CD3/anti-CD28 beads (Invitrogen) were used for polyclonal stimulation. After four days, proliferation of naïve $T$ cells was assessed by measuring $\mathrm{CTV}$ dilution in living naïve $\mathrm{CD} 4^{+} \mathrm{CD} 90.2^{+} \mathrm{CD} 45.1^{+} \mathrm{T}$ cells by flow cytometry.

\section{Allogeneic skin transplantation and adoptive transfer of cells}

To induce or prevent an immune reaction against the skin graft $2.5 \times 10^{5}$ FACS-sorted peripheral naïve T cells $\left(\mathrm{CD} 4^{+} \mathrm{CD} 90.2^{+} \mathrm{CD} 45.1^{+} \mathrm{CD} 25^{-} \mathrm{CD} 44^{-} \mathrm{CD} 6 \mathrm{~L}^{\mathrm{hi}}\right)$ isolated from female $\mathrm{C} 57 \mathrm{BL} / 6$ mice were injected intravenously (i.v.) into female Rag2 ${ }^{-/}$mice $(\mathrm{C} 57 \mathrm{BL} / 6)$ with or without $5 \times 10^{5}$ allo-iTregs $\left(\mathrm{CD} 4^{+} \mathrm{CD} 90.2^{+} \mathrm{Foxp}^{\mathrm{RFP}+}\right)$. As control, PBS was i.v injected into Rag2 $2^{-/}$mice. The following day mice were anesthetized with an equal mixture of 2 $\%$ Rompun (Bayer) and $100 \mathrm{mg} / \mathrm{ml} \mathrm{Ketanest} \mathrm{(Eurovet}$ Animal Health B.V., $500 \mu \mathrm{l}$ each) with $4.5 \mathrm{ml} \mathrm{NaCl} .10$ $\mu 1$ per $\mathrm{g}$ bodyweight were administered intraperitoneally (i.p.) into the mouse. Full thickness tail skin (about 1 $\mathrm{cm}^{2 ;} \mathrm{BALB} / \mathrm{c}$ ) was grafted onto the lateral flank of the recipient $\mathrm{Rag}^{-/-}$mouse. The wound was applied with an ointment and was protected firstly with an elastic bandage (Rancolast $^{\circledR}$, Lohmann\&Rauscher). Then a soft tape (Leukopor $^{\circledR}, \mathrm{BSN}$ ) was applied and finally the dressing was done using the surgical tape (Blenderm $\left.{ }^{\mathrm{TM}}, 3 \mathrm{M}\right)$. The whole bandage was carefully removed after 7-10 days. The graft was monitored every two days for rejection characteristics. Rapamycin (LC Laboratories) was injected i.p. three times at day -1 , day 0 and day 2 at $90 \mu \mathrm{g} / \mathrm{mouse}$. Mice were monitored for graft survival until day 100 .

\section{RNA-Seq}

Total RNA was isolated from purified APCs with RNAeasy Plus mini kit (Qiagen). Quality and integrity of total RNA was controlled on Agilent Technologies 2100 Bioanalyzer (Agilent Technologies). Purification of poly-A containing mRNA was done using poly-T oligo attached magnetic beads (Illumina). Following the purification, the mRNA was used for library preparation using Script Seq v2 Library preparation kit (Illumina). The sequencing was carried out on Illumina HiSeq2500 using $50 \mathrm{bp}$ single read. The sequenced libraries were assessed for read quality with FastQC (http://www. bioinformatics.babraham.ac.uk/projects/fastqc). Quality assessment showed neither insufficient read quality, nor nucleotide frequency biases introduced by primer contamination. Therefore, libraries were directly aligned versus mouse reference genome (assembly: GRCm38) using splice junction mapper Tophat2 v1.2.0 [71] with default parameterization. Supplementary Table S4 shows an overview of resulting mapping statistics.

Reads aligned to annotated genes were quantified with htseq-count (http://www-huber.embl.de/users/ anders/HTSeq) program, and determined read counts served as input to DESeq2 [72] for pairwise detection and quantification of differential gene expression between the three different conditions. In addition, RPKM (reads per kilobase max. transcript length per million mapped reads) values were computed for each library from raw gene counts and a PCA of the $\log _{2}$ transformed, scaled and mean centred RPKM values was performed using base functions scale and prcomp from the statistical data analysis framework $R$. Shown heatmaps were generated with $\mathrm{R}$ package pheatmap. The list of DESeq 2 determined differentially expressed genes (DEGs) was filtered with an absolute $\log _{2}$ fold change (FC) cutoff of at least 1.5 and a $p$-value cutoff, corrected for multiple testing, of at most 0.05 . This filtering results in the numbers of up-/ down-regulated genes shown in Supplementary Table S1. RNA-Seq data can be accessed under GEO/SRA accession number GSE67834.

\section{Gene ontology and pathway enrichment analysis}

The association of Gene Ontology (GO) terms and KEGG metabolic pathways to genes in the lists of DEGs $(|\log 2 \mathrm{FC}|>=1.5$ and adjusted $p$-value $<=0.05)$ resulting from the pairwise comparisons mTECs vs. sp-DCs, mTECs vs. t-DCs and t-DCs vs. sp-DCs was assessed with functions from the R package GOstats [73]. For the applied conditional hypergeometric test for overrepresentation of GO terms in each of the three ontologies (molecular function, biological process and cellular component) and annotated KEGG pathways we used a $p$-value cutoff of 0.001 . The used GO annotations were obtained from the Bioconductor Mus musculus annotation package, whereas KEGG pathway annotations were directly retrieved from KEGG using KEGG`s REST API. 


\section{Methylation analysis}

For all methylation analyses, cells from male mice were used. Genomic DNA was isolated from purified cells with the NucleoSpin Tissue XS kit (MachereyNagel) following the manufacturer's recommendations. Purified DNA was quantified by measuring the absorption of light at $260 \mathrm{~nm}$ wavelength with a Nanodrop 1000 spectrophotometer (Peqlab). For analysis of TSDR, the TSDR was amplified by PCR containing $10 \mathrm{ng}$ of bisulfite-converted genomic DNA, HotStar Taq PCR buffer (Qiagen), 1 U HotStar Taq DNA polymerase, 2.5 $\mathrm{mM} \mathrm{MgCl}{ }_{2}$ and $0.38 \mu \mathrm{M}$ each of forward and reverse primers in a final volume of $50 \mu \mathrm{l}$ (Cycle: $95^{\circ} \mathrm{C}$ for 15 $\min ; 50 \mathrm{x} 95^{\circ} \mathrm{C}$ for $30 \mathrm{sec}, 57^{\circ} \mathrm{C}$ for $1 \mathrm{~min}, 72^{\circ} \mathrm{C}$ for 1 $\min ; 72^{\circ} \mathrm{C}$ for $7 \mathrm{~min}$ ). The PCR product was analyzed by gel electrophoresis. 20-40 $\mu 1$ of the PCR product, Pyromark Gold Q96 reagents (Qiagen), Pyromark buffers (Qiagen), Streptavidin Sepharose (GE Healthcare) and sequencing primers (Supplementary Table S5) were used for pyrosequencing on a PSQ96MA (Qiagen) according to the manufacturer's protocol. For Treg-specific epigenetic signature genes, the analysis was carried out using bisulfite sequencing as described previously [13].

\section{Statistics}

Calculations were performed using Graph Pad Prism v5.0 (Graph-Pad software). Most of the analyses were performed using nonparametric Mann-Whitney test (two-tailed, confidence intervals $=95 \%$ ) to compare groups and calculate $p$-values. Survival curves were calculated using Kaplan-Meier analysis and the $p$-values were analyzed with log-rank test (Mantel-Cox). A $p$-value of $p<0.05$ was considered significant.

\section{Data and materials availability}

The RNA-Seq data of the present study can be found via the following private access link:

http://www.ncbi.nlm.nih.gov/geo/query/acc. cgi?token $=$ ozshogkqxrklfkz\&acc $=$ GSE67834 .

\section{Author contributions}

G.G., E.N., M.H.-W., A.T. and S.F. performed the experiments and interpreted the data. N.O. and S.S. interpreted the methylation data of Treg-specific epigenetic signature genes. R.G. generated RNA-Seq data. M.B. performed the RNA-Seq data analysis. T.M. and S.H. performed in vivo CD40-CD40L blocking experiments and interpreted the data. E.L., N.G. and A.O. kindly provided mice and discussed the data. E.J. interpreted the data from skin transplantation experiments and contributed to the manuscript. G.G. and J.H. designed the research, interpreted the data, and wrote the paper.

\section{ACKNOWLEDGMENTS}

We thank Lothar Groebe, Beate Pietzsch, Aya Kawasaki, Joern Pezoldt and Maren Sievers for cell sorting, bisulfite pyrosequencing for TSDR, bisulfite sequencing for Treg-specific epigenetic signature genes, visualization of RNA-Seq data and mice monitoring for in vivo skin transplant, respectively.

\section{CONFLICTS OF INTEREST}

The authors declare no competing financial interests.

\section{GRANT SUPPORT}

This work was supported by the Collaborative Research Centre 738 "Optimization of conventional and innovative transplants" of the German Research Foundation (to J.H., R.G. and E.J.).

\section{REFERENCES}

1. Sakaguchi S, Yamaguchi T, Nomura T, Ono M. Regulatory T cells and immune tolerance. Cell. 2008; 133:775-787.

2. Riley JL, June CH, Blazar BR. Human T regulatory cell therapy: take a billion or so and call me in the morning. Immunity. 2009; 30:656-665.

3. Edinger M, Hoffmann P, Ermann J, Drago K, Fathman $\mathrm{CG}$, Strober S, Negrin RS. CD $4{ }^{+} \mathrm{CD} 25^{+}$regulatory T cells preserve graft-versus-tumor activity while inhibiting graftversus-host disease after bone marrow transplantation. Nat Med. 2003; 9:1144-1150.

4. Hippen KL, Riley JL, June CH, Blazar BR. Clinical perspectives for regulatory $\mathrm{T}$ cells in transplantation tolerance. Semin Immunol. 2011; 23:462-468.

5. Lundsgaard D, Holm TL, Hornum L, Markholst H. In vivo control of diabetogenic T-cells by regulatory $\mathrm{CD} 4{ }^{+} \mathrm{CD} 25^{+}$ T-cells expressing Foxp3. Diabetes. 2005; 54:1040-1047.

6. Tsaur I, Gasser M, Aviles B, Lutz J, Lutz L, Grimm M, Lange V, Lopau K, Heemann U, Germer CT, Chandraker A, Waaga-Gasser AM. Donor antigen-specific regulatory T-cell function affects outcome in kidney transplant recipients. Kidney Int. 2011; 79:1005-1012.

7. Bestard O, Cruzado JM, Mestre M, Caldes A, Bas J, Carrera M, Torras J, Rama I, Moreso F, Seron D, Grinyo JM. Achieving donor-specific hyporesponsiveness is associated with $\mathrm{FOXP}^{+}$regulatory $\mathrm{T}$ cell recruitment in human renal allograft infiltrates. J Immunol. 2007; 179:4901-4909.

8. Edinger M, Hoffmann P. Regulatory T cells in stem cell transplantation: strategies and first clinical experiences. Curr Opin Immunol. 2011; 23:679-684. 
9. Chen W, Jin W, Hardegen N, Lei KJ, Li L, Marinos N, McGrady G, Wahl SM. Conversion of peripheral $\mathrm{CD} 4{ }^{+} \mathrm{CD} 25^{-}$naive $\mathrm{T}$ cells to $\mathrm{CD} 4{ }^{+} \mathrm{CD} 25^{+}$regulatory $\mathrm{T}$ cells by TGF-beta induction of transcription factor Foxp3. J Exp Med. 2003; 198:1875-1886.

10. Polansky JK, Kretschmer K, Freyer J, Floess S, Garbe A, Baron U, Olek S, Hamann A, von Boehmer H, Huehn J. DNA methylation controls Foxp3 gene expression. Eur J Immunol. 2008; 38:1654-1663.

11. Koenecke C, Czeloth N, Bubke A, Schmitz S, Kissenpfennig A, Malissen B, Huehn J, Ganser A, Forster R, Prinz I. Alloantigen-specific de novo-induced Foxp ${ }^{+}$ Treg revert in vivo and do not protect from experimental GVHD. Eur J Immunol. 2009; 39:3091-3096.

12. Sela U, Olds P, Park A, Schlesinger SJ, Steinman RM. Dendritic cells induce antigen-specific regulatory $\mathrm{T}$ cells that prevent graft versus host disease and persist in mice. $\mathrm{J}$ Exp Med. 2011; 208:2489-2496.

13. Ohkura N, Hamaguchi M, Morikawa H, Sugimura K, Tanaka A, Ito Y, Osaki M, Tanaka Y, Yamashita R, Nakano N, Huehn J, Fehling HJ, Sparwasser T, et al. T cell receptor stimulation-induced epigenetic changes and Foxp3 expression are independent and complementary events required for Treg cell development. Immunity. 2012; 37:785-799.

14. Safinia N, Vaikunthanathan T, Fraser H, Thirkell S, Lowe K, Blackmore L, Whitehouse G, Martinez-Llordella M, Jassem W, Sanchez-Fueyo A, Lechler RI, Lombardi G. Successful expansion of functional and stable regulatory $\mathrm{T}$ cells for immunotherapy in liver transplantation. Oncotarget. 2016; 7:7563-7577. doi: 10.18632/oncotarget.6927.

15. Floess S, Freyer J, Siewert C, Baron U, Olek S, Polansky J, Schlawe K, Chang HD, Bopp T, Schmitt E, Klein-Hessling S, Serfling E, Hamann A, Huehn J. Epigenetic control of the foxp3 locus in regulatory T cells. PLoS Biol. 2007; 5:e38.

16. Huehn J, Polansky JK, Hamann A. Epigenetic control of FOXP3 expression: the key to a stable regulatory T-cell lineage? Nat Rev Immunol. 2009; 9:83-89.

17. Zheng Y, Josefowicz S, Chaudhry A, Peng XP, Forbush $\mathrm{K}$, Rudensky AY. Role of conserved non-coding DNA elements in the Foxp3 gene in regulatory T-cell fate. Nature. 2010; 463:808-812.

18. Ohkura N, Kitagawa Y, Sakaguchi S. Development and maintenance of regulatory T cells. Immunity. 2013; 38:414423.

19. Morikawa H, Ohkura N, Vandenbon A, Itoh M, NagaoSato S, Kawaji H, Lassmann T, Carninci P, Hayashizaki Y, Forrest AR, Standley DM, Date H, Sakaguchi S, Consortium F. Differential roles of epigenetic changes and Foxp3 expression in regulatory $\mathrm{T}$ cell-specific transcriptional regulation. Proc Natl Acad Sci U S A. 2014; 111:5289-5294.

20. Morikawa H and Sakaguchi S. Genetic, epigenetic basis of Treg cell development and function: from a FoxP3-centered view to an epigenome-defined view of natural Treg cells. Immunol Rev. 2014; 259:192-205.

21. Toker A, Engelbert D, Garg G, Polansky JK, Floess S, Miyao T, Baron U, Duber S, Geffers R, Giehr P, Schallenberg S, Kretschmer K, Olek S, et al. Active demethylation of the Foxp3 locus leads to the generation of stable regulatory $\mathrm{T}$ cells within the thymus. J Immunol. 2013; 190:3180-3188.

22. Kitagawa Y, Ohkura N, Sakaguchi S. Epigenetic control of thymic Treg-cell development. Eur J Immunol. 2015; 45:11-16.

23. Jordan MS, Boesteanu A, Reed AJ, Petrone AL, Holenbeck AE, Lerman MA, Naji A, Caton AJ. Thymic selection of $\mathrm{CD} 4{ }^{+} \mathrm{CD} 25^{+}$regulatory $\mathrm{T}$ cells induced by an agonist selfpeptide. Nat Immunol. 2001; 2:301-306.

24. Apostolou I, Sarukhan A, Klein L, von Boehmer H. Origin of regulatory $\mathrm{T}$ cells with known specificity for antigen. Nat Immunol. 2002; 3:756-763.

25. Fontenot JD, Rasmussen JP, Williams LM, Dooley JL, Farr AG, Rudensky AY. Regulatory T cell lineage specification by the forkhead transcription factor foxp3. Immunity. 2005; 22:329-341.

26. Tai X, Cowan M, Feigenbaum L, Singer A. CD28 costimulation of developing thymocytes induces Foxp3 expression and regulatory $\mathrm{T}$ cell differentiation independently of interleukin 2. Nat Immunol. 2005; 6:152162.

27. Aschenbrenner K, D'Cruz LM, Vollmann EH, Hinterberger M, Emmerich J, Swee LK, Rolink A, Klein L. Selection of Foxp3+ regulatory $\mathrm{T}$ cells specific for self antigen expressed and presented by Aire ${ }^{+}$medullary thymic epithelial cells. Nat Immunol. 2007; 8:351-358.

28. Proietto AI, van Dommelen S, Zhou P, Rizzitelli A, D'Amico A, Steptoe RJ, Naik SH, Lahoud MH, Liu Y, Zheng P, Shortman K, Wu L. Dendritic cells in the thymus contribute to T-regulatory cell induction. Proc Natl Acad Sci U S A. 2008; 105:19869-19874.

29. Lio CW, Hsieh CS. A two-step process for thymic regulatory $\mathrm{T}$ cell development. Immunity. 2008; 28:100111.

30. Burchill MA, Yang J, Vang KB, Moon JJ, Chu HH, Lio CW, Vegoe AL, Hsieh CS, Jenkins MK, Farrar MA. Linked $\mathrm{T}$ cell receptor and cytokine signaling govern the development of the regulatory $\mathrm{T}$ cell repertoire. Immunity. 2008; 28:112-121.

31. Spence PJ, Green EA. Foxp $3^{+}$regulatory $T$ cells promiscuously accept thymic signals critical for their development. Proc Natl Acad Sci U S A. 2008; 105:973978.

32. Lee HM, Hsieh CS. Rare development of Foxp3 ${ }^{+}$ thymocytes in the $\mathrm{CD}^{+} \mathrm{CD}^{+}$subset. J Immunol. 2009; 183:2261-2266.

33. Lee HM, Bautista JL, Scott-Browne J, Mohan JF, Hsieh CS. A Broad Range of Self-Reactivity Drives Thymic 
Regulatory T Cell Selection to Limit Responses to Self. Immunity. 2012; 37:475-486.

34. Coquet JM, Ribot JC, Babala N, Middendorp S, van der Horst G, Xiao Y, Neves JF, Fonseca-Pereira D, Jacobs $\mathrm{H}$, Pennington DJ, Silva-Santos B, Borst J. Epithelial and dendritic cells in the thymic medulla promote $\mathrm{CD} 4^{+} \mathrm{Foxp} 3^{+}$ regulatory $\mathrm{T}$ cell development via the $\mathrm{CD} 27-\mathrm{CD} 70$ pathway. J Exp Med. 2013; 210:715-728.

35. Oh J, Wu N, Baravalle G, Cohn B, Ma J, Lo B, Mellman I, Ishido S, Anderson M, Shin JS. MARCH1-mediated MHCII ubiquitination promotes dendritic cell selection of natural regulatory T cells. J Exp Med. 2013; 210:10691077.

36. Metzger TC, Khan IS, Gardner JM, Mouchess ML, Johannes KP, Krawisz AK, Skrzypczynska KM, Anderson MS. Lineage tracing and cell ablation identify a post-Aireexpressing thymic epithelial cell population. Cell reports. 2013; 5:166-179.

37. Cowan JE, Parnell SM, Nakamura K, Caamano JH, Lane PJ, Jenkinson EJ, Jenkinson WE, Anderson G. The thymic medulla is required for Foxp $3^{+}$regulatory but not conventional CD4+ thymocyte development. J Exp Med. 2013; 210:675-681.

38. Wirnsberger G, Mair F, Klein L. Regulatory T cell differentiation of thymocytes does not require a dedicated antigen-presenting cell but is under $\mathrm{T}$ cell-intrinsic developmental control. Proc Natl Acad Sci U S A. 2009; 106:10278-10283.

39. Ma DY, Clark EA. The role of CD40 and CD154/CD40L in dendritic cells. Semin Immunol. 2009; 21:265-272.

40. Miyao T, Floess S, Setoguchi R, Luche H, Fehling HJ, Waldmann H, Huehn J, Hori S. Plasticity of Foxp $3^{+}$T cells reflects promiscuous Foxp3 expression in conventional $\mathrm{T}$ cells but not reprogramming of regulatory $\mathrm{T}$ cells. Immunity. 2012; 36:262-275.

41. Josefowicz SZ, Lu LF, Rudensky AY. Regulatory T cells: mechanisms of differentiation and function. Annu Rev Immunol. 2012; 30:531-564.

42. Huehn J, Hamann A. Homing to suppress: address codes for Treg migration. Trends Immunol. 2005; 26:632-636.

43. Nadig SN, Wieckiewicz J, Wu DC, Warnecke G, Zhang W, Luo S, Schiopu A, Taggart DP, Wood KJ. In vivo prevention of transplant arteriosclerosis by ex vivo-expanded human regulatory T cells. Nat Med. 2010; 16:809-813.

44. Ferrer IR, Hester J, Bushell A, Wood KJ. Induction of transplantation tolerance through regulatory cells: from mice to men. Immunol Rev. 2014; 258:102-116.

45. Brunstein CG, Miller JS, Cao Q, McKenna DH, Hippen KL, Curtsinger J, Defor T, Levine BL, June CH, Rubinstein P, McGlave PB, Blazar BR, Wagner JE. Infusion of ex vivo expanded $\mathrm{T}$ regulatory cells in adults transplanted with umbilical cord blood: safety profile and detection kinetics. Blood. 2011; 117:1061-1070.

46. Feng G, Gao W, Strom TB, Oukka M, Francis RS, Wood
KJ, Bushell A. Exogenous IFN-gamma ex vivo shapes the alloreactive T-cell repertoire by inhibition of Th17 responses and generation of functional Foxp $3^{+}$regulatory T cells. Eur J Immunol. 2008; 38:2512-2527.

47. Sagoo P, Ali N, Garg G, Nestle FO, Lechler RI, Lombardi G. Human regulatory $\mathrm{T}$ cells with alloantigen specificity are more potent inhibitors of alloimmune skin graft damage than polyclonal regulatory T cells. Sci Transl Med. 2011; 3:83ra42.

48. Moore C, Tejon G, Fuentes C, Hidalgo Y, Bono MR, Maldonado P, Fernandez R, Wood KJ, Fierro JA, Rosemblatt M, Sauma D, Bushell A. Alloreactive regulatory $\mathrm{T}$ cells generated with retinoic acid prevent skin allograft rejection. Eur J Immunol. 2015; 45:452-463.

49. Allan SE, Crome SQ, Crellin NK, Passerini L, Steiner TS, Bacchetta R, Roncarolo MG, Levings MK. Activationinduced FOXP3 in human T effector cells does not suppress proliferation or cytokine production. Int Immunol. 2007; 19:345-354.

50. Campos-Mora M, Morales RA, Perez F, Gajardo T, Campos J, Catalan D, Aguillon JC, Pino-Lagos K. Neuropilin- $1^{+}$ regulatory $\mathrm{T}$ cells promote skin allograft survival and modulate effector $\mathrm{CD}^{+} \mathrm{T}$ cells phenotypic signature. Immunol Cell Biol. 2015; 93:113-119.

51. Dijke IE, Hoeppli RE, Ellis T, Pearcey J, Huang Q, McMurchy AN, Boer K, Peeters AM, Aubert G, Larsen I, Ross DB, Rebeyka I, Campbell A, et al. Discarded Human Thymus Is a Novel Source of Stable and Long-Lived Therapeutic Regulatory T Cells. Am J Transplant. 2016; 16:58-71.

52. Sharma MD, Huang L, Choi JH, Lee EJ, Wilson JM, Lemos H, Pan F, Blazar BR, Pardoll DM, Mellor AL, Shi H, Munn DH. An inherently bifunctional subset of Foxp $3^{+} \mathrm{T}$ helper cells is controlled by the transcription factor eos. Immunity. 2013; 38:998-1012.

53. Pan F, Yu H, Dang EV, Barbi J, Pan X, Grosso JF, Jinasena D, Sharma SM, McCadden EM, Getnet D, Drake CG, Liu JO, Ostrowski MC, Pardoll DM. Eos mediates Foxp3dependent gene silencing in $\mathrm{CD}^{+}$regulatory $\mathrm{T}$ cells. Science. 2009; 325:1142-1146.

54. Wing K, Onishi Y, Prieto-Martin P, Yamaguchi T, Miyara M, Fehervari Z, Nomura T, Sakaguchi S. CTLA-4 control over Foxp $3^{+}$regulatory T cell function. Science. 2008; 322:271-275.

55. Mahmud SA, Manlove LS, Schmitz HM, Xing Y, Wang Y, Owen DL, Schenkel JM, Boomer JS, Green JM, Yagita H, Chi H, Hogquist KA, Farrar MA. Costimulation via the tumor-necrosis factor receptor superfamily couples TCR signal strength to the thymic differentiation of regulatory $\mathrm{T}$ cells. Nat Immunol. 2014; 15:473-481.

56. Lin J, Yang L, Silva HM, Trzeciak A, Choi Y, Schwab SR, Dustin ML, Lafaille JJ. Increased generation of Foxp3 ${ }^{+}$ regulatory $\mathrm{T}$ cells by manipulating antigen presentation in the thymus. Nat Commun. 2016; 7:10562. 
57. Perry JS, Lio CW, Kau AL, Nutsch K, Yang Z, Gordon JI, Murphy KM, Hsieh CS. Distinct contributions of Aire and antigen-presenting-cell subsets to the generation of selftolerance in the thymus. Immunity. 2014; 41:414-426.

58. Kingsley CI, Karim M, Bushell AR, Wood KJ. CD25 $5^{+} \mathrm{CD} 4^{+}$ Regulatory T Cells Prevent Graft Rejection: CTLA-4- and IL-10-Dependent Immunoregulation of Alloresponses. The Journal of Immunology. 2002; 168:1080-1086.

59. Sawitzki B, Kingsley CI, Oliveira V, Karim M, Herber M, Wood KJ. IFN-gamma production by alloantigen-reactive regulatory $\mathrm{T}$ cells is important for their regulatory function in vivo. J Exp Med. 2005; 201:1925-1935.

60. Koenecke C, Lee CW, Thamm K, Fohse L, Schafferus M, Mittrucker HW, Floess S, Huehn J, Ganser A, Forster R, Prinz I. IFN-gamma production by allogeneic Foxp ${ }^{+}$ regulatory $\mathrm{T}$ cells is essential for preventing experimental graft-versus-host disease. J Immunol. 2012; 189:2890-2896.

61. Koch MA, Tucker-Heard G, Perdue NR, Killebrew JR, Urdahl KB, Campbell DJ. The transcription factor T-bet controls regulatory $\mathrm{T}$ cell homeostasis and function during type 1 inflammation. Nat Immunol. 2009; 10:595-602.

62. Hasegawa H, Inoue A, Kohno M, Lei J, Miyazaki T, Yoshie O, Nose M, Yasukawa M. Therapeutic effect of CXCR3expressing regulatory $\mathrm{T}$ cells on liver, lung and intestinal damages in a murine acute GVHD model. Gene Ther. 2008; 15:171-182.

63. Derbinski J, Schulte A, Kyewski B, Klein L. Promiscuous gene expression in medullary thymic epithelial cells mirrors the peripheral self. Nat Immunol. 2001; 2:1032-1039.

64. Li J, Park J, Foss D, Goldschneider I. Thymus-homing peripheral dendritic cells constitute two of the three major subsets of dendritic cells in the steady-state thymus. J Exp Med. 2009; 206:607-622.

65. Cuss SM, Green EA. Abrogation of CD40-CD154 signaling impedes the homeostasis of thymic resident regulatory $\mathrm{T}$ cells by altering the levels of IL-2, but does not affect regulatory T cell development. J Immunol. 2012; 189:17171725 .
66. Wan YY, Flavell RA. Identifying Foxp3-expressing suppressor T cells with a bicistronic reporter. Proc Natl Acad Sci U S A. 2005; 102:5126-5131.

67. Kamanaka M, Kim ST, Wan YY, Sutterwala FS, LaraTejero M, Galan JE, Harhaj E, Flavell RA. Expression of interleukin-10 in intestinal lymphocytes detected by an interleukin-10 reporter knockin tiger mouse. Immunity. 2006; 25:941-952.

68. Caton ML, Smith-Raska MR, Reizis B. Notch-RBP-J signaling controls the homeostasis of CD8- dendritic cells in the spleen. J Exp Med. 2007; 204:1653-1664.

69. Wolenski M, Cramer SO, Ehrlich S, Steeg C, Fleischer B, von Bonin A. Enhanced activation of CD83-positive T cells. Scand J Immunol. 2003; 58:306-311.

70. Garcia-Martinez LF, Appleby MW, Staehling-Hampton K, Andrews DM, Chen Y, McEuen M, Tang P, Rhinehart RL, Proll S, Paeper B, Brunkow ME, Grandea AG 3rd, Howard ED, et al. A novel mutation in CD83 results in the development of a unique population of $\mathrm{CD}^{+} \mathrm{T}$ cells. $\mathrm{J}$ Immunol. 2004; 173:2995-3001.

71. Kim D, Pertea G, Trapnell C, Pimentel H, Kelley R, Salzberg SL. TopHat2: accurate alignment of transcriptomes in the presence of insertions, deletions and gene fusions. Genome Biol. 2013; 14:R36.

72. Love MI, Huber W, Anders S. Moderated estimation of fold change and dispersion for RNA-seq data with DESeq2. Genome Biol. 2014; 15:550.

73. Falcon S, Gentleman R. Using GOstats to test gene lists for GO term association. Bioinformatics. 2007; 23:257-258. 